Pacific Journal of Mathematics

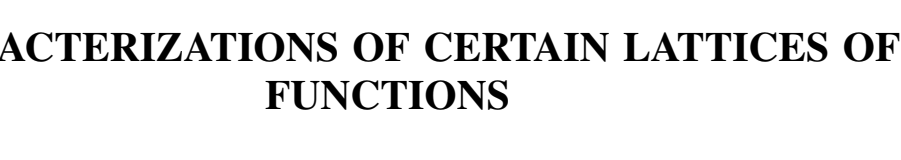




\title{
CHARACTERIZATIONS OF CERTAIN LATTICES OF FUNCTIONS
}

\author{
Frank W. Anderson and Robert L. Blair
}

Introduction. The set $C(X, R)$ of all real-valued continuous functions on a compact Hausdorff space $X$ has been characterized from a variety of points of view. We mention, in particular, those characterizations of $C(X, R)$ as a partially ordered system of some prescribed kind: namely, the characterizations of $C(X, R)$ by Stone as a partially ordered ring [14] and as a lattice-ordered group [15], those by Kakutani [7] and by M. and S. Krein [11] as a lattice-ordered Banach space, and those by Fan [4] and Fleischer [5] as a partially ordered group. The problem of characterizing $C(X, R)$ as a lattice alone was posed by Birkhoff [1, Problem 81] and by Kaplansky [9]. As a partial solution of this problem Kaplansky [9] characterized certain sublattices of $C(X, R)$ as "translation lattices". A solution of the general problem has recently been obtained by Heider [6], and, still more recently, another solution has been announced by Pinsker [12].

In the present paper we obtain, as corollaries of our main results, two new characterizations of the lattice $C(X, R)$. We shall actually solve, however, problems somewhat more general than that of Birkhoff and Kaplansky mentioned above. In the first place, we replace the real chain $R$ by a conditionally complete dense-in-itself chain $K$ which has neither a first nor a last element and which is equipped with its interval topology. In the second, we characterize not only $C(X, K)$ but also an extensive class of sublattices of $C(X, K)$.

We give next a more detailed summary of the results of this paper; following this, we pose some unsolved problems suggested by these results.

A sublattice $L$ of $C(X, K)$ is characterizing (Definition 1.1) in case $L$ separates points in $X$ in a certain strong sense. The space $X$ is $K$ normal in case $C(X, K)$ is itself characterizing. In Definition 2.10 the notion of an " $S$-lattice" is introduced. The main result (Theorem 2.16) of $\S 2$ states that a characterizing sublattice of $C(X, K)$ is an $S$-lattice. (This usage of the term "S-lattice" is inexact but will suffice for the present; the concept itself is inspired by work of Shirota [13].) Section 3 is devoted to a further study of $S$-lattices and of "S-ideals" in $S$ lattices. The results of $\S 3$, when applied (in $\S 4$ ) to a characterizing sublattice $L$ of $C(X, K)$, enable us to reconstruct $X$ as a space of

Received August 18, 1958. The major portion of this work was completed in the summer of 1956 at which time the first named author held a grant from the University of Nebraska Research Council. Presented to the American Mathematical Society, August 27, 1957. 
maximal $S$-ideals of $L$. From this it follows (Theorem 4.3) that $L$ completely determines the topology of $X$.

Those characterizing sublattices of $C(X, K)$ which contain a set $Q$ of constant functions order-dense in $K$ constitute an extensive and reasonably accessible subclass of the class of all characterizing sublattices of $C(X, K)$; we call such sublattices characterizing $Q$-sublattices of $C(X, K)$. This notion finds its abstract counterpart in the concept of a " $C_{1}$-lattice relative to a separating chain $Q$ " (Definition 5.8). The main result (Theorem 5.12) of $\S 5$ states that a characterizing $Q$-sublattice of $C(X, K)$ is a $C_{1}$-lattice relative to $Q$.

Section 6 is devoted to " $C$-lattices" (Definition 5.8), a somewhat wider class of lattices than that of $C_{1}$-lattices. Theorem 6.8 asserts that a $C$-lattice $L$ is also an $S$-lattice so that the results of $\S 3$ are applicable. It then becomes possible to associate with $L$ a uniquely determined compact Hausdorff space $X_{L}$ (Theorem 6.16).

In $\S 7$ we are ready to attack the problem of representing a bounded $C$-lattice (Definition 7.3) as a lattice of functions. As a preliminary result, Theorem 7.4 states (in effect) that a bounded $C$-lattice admits a characterizing $Q$-sublattice of $C\left(X_{L}, K\right)$ as a homomorphic image. Theorem 7.7 then accomplishes a complete description of characterizing $Q$-sublattices: A lattice $L$ is isomorphic to a characterizing $Q$-sublattice of $C(X, K)$ for some (topologically unique) compact $K$-normal space $X$ if and only if $L$ is a bounded $C_{1}$-lattice relative to $Q$. Once this basic result is at hand, one need only impose on the bounded $C_{1}$-lattice $L$ a suitable hypothesis of completeness in order to obtain the entire lattice $C(X, K)$. This we do in $\S 8$ in two different ways: In the first, an intrinsically defined uniformity is introduced on $L$ and $L$ is required to be locally complete in this uniformity (Theorem 8.3); in the second, Fan's notion of a direct extension [4] is adapted to the present context and $L$ is required to be isomorphic to each of its bounded direct extensions (Theorem 8.9). Solutions of the Birkhoff-Kaplansky problem emerge as Corollaries 8.4 and 8.10 .

In the concluding section the results of the present paper are compared with certain earlier characterizations of $C(X, R)$. In particular, we indicate how our results can be used to deduce the characterizations of Stone, Fan, and Fleischer mentioned above.

The results of the present paper suggest certain more general problems.

I. Given an arbitrary chain $K$, characterize those lattices $L$ which are isomorphic to $C(X, K)$ for some (compact) space $X$.

II. Given an arbitrary chain $K$, characterize those lattices $L$ with the property that, for some (compact) space $X, L$ is isomorphic to some 
sublattice of $C(X, K)$ which determines $X$. In particular, characterize those lattices $L$ with the property that, for some compact space $X, L$ is isomorphic to some sublattice of $C(X, K)$ which is characterizing in the sense of Definition 1.1.

Related problems are obtained from problems I and II by dropping the requirement that the chain $K$ be specified in advance.

The difficulty involved in solving either of these problems will probably depend upon the type of solution sought. In this connection it is clear that the type of solution obtained is of an importance approaching that of the solution itself. We propose, as the most desirable type of solution, that type in which the conditions imposed on the lattice $\langle L, \mathrm{~V}, \mathbf{\Lambda}\rangle$ are all arithmetical [17] relative to $\langle L, \mathrm{~V}, \Lambda\rangle$; that is, formulable solely in terms of (i) elements of $L$, (ii) elementary logical constants (connectives, quantifiers, identity symbol), and (iii) the operations $V$ and $\Lambda$. (Thus, for example, conditions involving assertions about ideals of $L$ are non-arithmetical and, therefore, in such a solution, inadmissable.) A solution of either I or II of this ideal type is probably impossible. In the first place, a (non-arithmetical) assumption of the sort that $L$ contains (a replica of) a suitable subset $Q$ of $K$ seems inevitable. Admitting this assumption, however, and introducing the predicate $\mathscr{E}_{Q}$ expressing elementhood in $Q$, we can still insist that allowable conditions be arithmetical relative to the system $\left\langle L, \bigvee, \Lambda, \mathscr{E}_{Q}\right\rangle$; that is, formulable solely in terms of (i)-(iii) and (iv) the predicate $\mathscr{E}_{Q}$. A solution of II of this modified type seems not unfeasible. When it comes to I, however, a solution seems to require first a solution of II (in some form) followed by the imposition of a suitable hypothesis of completeness. Again, it appears difficult, if not impossible, to formulate such a completeness hypothesis arithmetically relative to $\left\langle L, \mathrm{~V}, \Lambda, \mathscr{E}_{Q}\right\rangle$. For a complete solution of I, therefore, a further relaxation of arithmetical requirements seems unavoidable.

Using the above terminology we can now describe more precisely the nature of our present results and their relation to those of Heider and of Pinsker. Theorem 7.7, described above, is a solution of the special case of II in which $K$ is conditionally complete, dense-in-itself, and without extreme points, and in which the characterizing sublattice in question is a $Q$-sublattice. Moreover, our solution of this problem is arithmetical relative to $\left\langle L, \mathrm{~V}, \Lambda, \mathscr{E}_{Q}\right\rangle$. Theorems 8.3 and 8.9 are solutions of I with the specified restrictions on $K$; they are, furthermore, non-arithmetical (relative to $\left\langle L, \mathrm{~V}, \Lambda, \mathscr{E}_{Q}\right\rangle$ ) only in their hypotheses of completeness. These same remarks apply also to the solutions of the Birkhoff-Kaplansky problem embodied in Corollaries 8.4 and 8.10. On the other hand, an inspection of the solutions by Heider and by Pinsker of the Birkhoff-Kaplansky problem shows that they are both non-arithme- 
tical, not only with respect to their completeness hypotheses, but also with respect to most of their remaining hypotheses. We mention, in particular, that Heider relies on assumptions concerning the real lattice homomorphisms of $L$ and that (among other non-arithmetical assumptions) Pinsker assumes outright the existence of a certain dual lattice automorphism of $L$.

1. Preliminary remarks. In this section we list some of the definitions, notational conventions, and facts essential to the rest of the paper.

We begin by adopting the following convention: Throughout the paper $K$ will denote a chain which is conditionally complete [1], densein-itself [1], and with neither a first nor a last element.

The letter $R$ will be reserved for the chain of real numbers.

If $Q$ is any chain, then we shall denote by $\bar{Q}$ the completion of $Q$ by cuts $[1$, p. 58]. We denote by $\hat{Q}$ the conditionally complete subchain of $\bar{Q}$ obtained by removing those extreme points of $\bar{Q}$, if any, which are not in $Q$, and we call $\hat{Q}$ the conditional completion of $Q$. If $Q$ is an order-dense subchain [1] of $K$, then $\hat{Q}=K$ and $\bar{Q}=\bar{K}$.

If $Q$ is a chain and if $X$ is a non-empty set, then we denote by $F(X, Q)$ the set of all functions on $X$ to $Q$. For $f, g \in F(X, Q)$ we set $f \leqq g$ in case $f(x) \leqq g(x)$ for all $x \in X$; with respect to this partial ordering $F(X, Q)$ is a distributive lattice. If $A \subseteq X$ and if $f, g \in F(X, Q)$, then we shall say that " $f<g$ (respectively, $f \leqq g$ ) on $A$ " in case $f(x)<g(x)$ (respectively, $f(x) \leqq g(x)$ ) for all $x \in A$.

If $X$ is a topological space, and if $Q$ is endowed with its interval topology $[1$, p. 60], then we denote by $C(X, Q)$ the sublattice of $F(X, Q)$ consisting of all continuous functions on $X$ to $Q$. If $\alpha \in Q$, then we shall also denote by $\alpha$ the function in $C(X, Q)$ which is identically equal to $\alpha$ on $X$.

Let $X$ be a topological space. We shall for the most part be concerned with a certain class of sublattices of $C(X, K)$ which we now define.

DeFINITION 1.1. A sublattice $L$ of $C(X, K)$ is characterizing ${ }^{1}$ in case for each pair of distinct points $x, y$ in $X$ and each pair of functions $f, g$ in $L$, there is an $h \in L$ such that $h(x)<f(x)$ and $h(y)>g(y)$.

The proof of the following proposition will be omitted.

(1.2) If $X$ is compact and if $L$ is a characterizing sublattice of $C(X, K)$, then for each pair of disjoint closed subsets $A, B$ of $X$ and each pair $f, g \in L$ there is an $h \in L$ such that $h<f$ on $A$ and $h>g$ on $B$.

${ }^{1}$ If $X$ is compact, then, in view of our restrictions on $K$, this is equivalent to the notion of characterizing used in $[\mathbf{3}]$. 
For some compact Hausdorff spaces $X$ and some chains $K$ it may be the case that no sublattice of $C(X, K)$ is characterizing, and it may be the case that $C(X, K)$ itself is not characterizing even though it has characterizing sublattices.

Definition 1.3. We say that a compact space $X$ is $K$-normal in case $C(X, K)$ is characterizing $(c f .[8])$.

We observe that $K$-normal spaces do exist. In fact, every totally disconnected compact Hausdorff space is clearly $K$-normal. It is also clear that every compact $K$-normal space is necessarily Hausdorff.

Throughout the remainder of this paper we shall assume that $X$ is a compact Hausdorff space containing at least two points.

Definition 1.4. Let $Q$ be an order-dense subchain of $K$. Then a sublattice $L$ of $C(X, K)$ is a $Q$-sublattice of $C(X, K)$ in case $L$ contains the set $Q$ of constant functions.

We observe that if $Q_{1}$ is order-dense in $Q$ and if $Q$ is order-dense in $K$, then a $Q$-sublattice of $C(X, K)$ is also a $Q_{1}$-sublattice of $C(X, K)$ Note also that $C(X, K)$ contains a characterizing $Q$-sublattice if and only if $X$ is $K$-normal.

The symbols $U$ and $\cap$ will denote set union and intersection, respectively, whereas, when dealing with "abstract" lattices, the symbols $V$ and $\Lambda$ will be used to denote joins and meets.

If $A \subseteq X$, then we denote by $A^{-}$and $A^{\prime}$, respectively, the closure and the complement of $A$ in $X$.

2. A class of binary relations on characterizing sublattices. If $L$ is a distributive lattice, if $l \in L$, and if $\ll$ is a transitive binary , relation on $L$, then (according to Definition 2.10) $L$ is an $S$-lattice at $l$, relative to $\ll$, in case $L$ satisfies conditions $(2.11)-(2.15)$ below. The main purpose of this section is to prove that if $L$ is a characterizing sublattice of $C(X, K)$, and if $l \in L$, then there is a transitive binary relation $<_{l}$ on $L$ relative to which $L$ is an $S$-lattice at $l$. We point out that the results of this and of the next section rely heavily on the ideas of Shirota [13].

Definition 2.1. If $L$ is a distributive lattice and if $l \in L$, then we define the binary relation $\subset_{l}$ on $L$ as follows: For each pair $f, g \in L, f \subset_{l} g$ in case $h \wedge g \leqq l$ implies $h \wedge f \leqq l$ for every $h \in L$.

The following facts are easily verified.

(2.2) The relation $\subset_{l}$ is transitive on $L$.

(2.3) If $f, g \in L$ with $f \leqq g$, then $f \subset_{l} g$ for every $l \in L$.

(2.4) If $f \subset_{l} l$, then $f \leqq l$.

(2.5) If $f_{i}, g_{i} \in L$ with $f_{i} \subset_{l} g_{i}(i=1,2)$, then $f_{1} \wedge f_{2} \subset_{l} g_{1} \wedge g_{2}$ and $f_{1} \vee f_{2} \subset_{l} g_{1} \vee g_{2}$. 
Definition 2.6. If $L$ is a sublattice of $C(X, K)$, and if $l \in L$, then we define the binary relation $\kappa_{l}$ on $L$ as follows: ${ }^{2}$ For each pair $f, g \in L, f \ll_{l} g$ in case for each $k \in L$ there exist $g^{\prime}, h \in L$ such that $g^{\prime} \subset{ }_{l} g$ and such that both $g^{\prime} \vee h \geqq k$ and $f \wedge h \leqq l$.

It is clear (taking $g^{\prime}=g$ and $h=k$ ) that $l \ll_{l} g$ for every $g \in L$.

(2.7) Let $L$ be a sublattice of $C(X, K)$ and let $l \in L$. Then

(i) $\ll_{l}$ is transitive on $L$;

(ii) if $f, g \in L$ with $f \ll_{l} g$, then $f \subset_{l} g$.

Proof. The first statement follows at once from (ii) and the definition of $\ll_{l}$. Thus we shall prove (ii). Let $f \ll_{l} g$; then there exist $g^{\prime}, h \in L$ such that $g^{\prime} \subset_{l} g, g^{\prime} \vee h \geqq f$, and $f \wedge h \leqq l$. If $k \in L$ with $k \wedge g \leqq l$, then $k \wedge f=k \wedge\left(g^{\prime} \vee h\right) \wedge f=\left[\left(k \wedge g^{\prime}\right) \vee(k \wedge h)\right] \wedge f \leqq$ $[l \vee(k \wedge h)] \wedge f \leqq l \vee[k \wedge h \wedge f]=l$. Hence $f \subset_{l} g$.

If $f, g \in C(X, K)$, then we set

$$
P(f, g)=\{x \in X ; f(x)>g(x)\} .
$$

Observe that since $f$ and $g$ are continuous, $P(f, g)$ is open in $X$.

We now characterize the relations $c_{l}$ and $<_{l}$ on a characterizing sublattice in terms of sets of the form $P(f, l) .^{3}$

(2.8) If $L$ is a characterizing sublattice of $C(X, K)$, and if $f, g, l \in L$, then the following statements hold:

(i) $f \subset_{l} g$ if and only if $P(f, l)^{-} \subseteq P(g, l)^{-}$.

(ii) $f \ll_{l} g$ if and only if $P(f, l)^{-} \subseteq P(g, l)^{--1}$.

Proof. Statement (i) follows from (1.2) and the fact that if $f, h \in L$, then $P(f, l) \cap P(h, l)=P(f \wedge h, l)$.

To prove the second statement assume first that $f \ll_{l} g$. From (1.2) we conclude that $P(k, l)=X$ for some $k \in L$. Then there exist $g^{\prime}, h \in L$ such that $g^{\prime} \subset_{l} g, g^{\prime} \vee h \geqq k$, and $f \wedge h \leqq l$. Now if $x \in P(g, l)^{-1-}$, then $g^{\prime}(x) \leqq l(x)$, and hence $h(x) \geqq k(x)>l(x)$. Therefore $x \in P(h, l)$, and since $P(h, l) \cap P(f, l)=\phi$, we have $x \notin P(f, l)^{-}$.

Conversely, let $P(f, l)^{-} \subseteq P(g, l)^{-1-1}$ and let $k \in L$. By (1.2) there exist $g^{\prime}, h \in L$ such that $g^{\prime}>k$ on $P(f, l)^{-}, g^{\prime}<l$ on $P(g, l)^{-1-}, h<l$ on $P(f, l)^{-}$and $h>k$ on $P\left(g^{\prime}, k\right)^{\prime}$. Then $g^{\prime} \subset_{l} g, g^{\prime} \vee h \geqq k$, and $f \wedge h \leqq l$. That is, $f \ll_{l} g$.

Definition 2.9. If $L$ is any lattice and if $\rho$ is a binary relation on $L$, then an element $e \in L$ is a unit for $\rho$ in case $f \rho e$ for every $f \in L$.

${ }^{2}$ If $L$ has a least element $l$, then it can be shown that the relation $\ll_{l}$ coincides with the relation $\ll$ of Shirota [13, Definition 4]. We note, however, that if $L$ is characterizing, then $L$ has no least element.

${ }^{3}$ Cf. [13, Lemma 1]. 
If $L$ is a distributive lattice and if $l \in L$, then we denote by $E_{l}$ the set of all units of $L$ for the binary relation $\subset_{l}$.

We note that if $L$ is a characterizing sublattice of $C(X, K)$ and if $l \in L$, then by (2.8) and (1.2) it is clear that $f \in E_{l}$ if and only if $P(f, l)^{-}=X$.

Definition 2.10. Let $L$ be a distributive lattice, let $l \in L$, and let $\ll$ be a transitive binary relation on $L$. We say that $L$ is an $S$-lattice at $l$ (relative to $\ll$ ) in case the following conditions are satisfied: ${ }^{4}$

(2.11) If $f_{i} \ll g_{i}(i=1,2)$, then $f_{1} \vee f_{2} \ll g_{1} \vee g_{2}$.

(2.12) If $f \ll g$, then $f \ll h \ll g$ for some $h \in L$.

(2.13) $L$ contains a unit for $\ll$.

(2.14) If $f \ll g$, then $f \wedge h \leqq l$ and $g \vee h \in E_{l}$ for some $h \in L$.

(2.15) If $f \ll g$, if $g \wedge k \leqq l$, and if $f \vee h \in E_{l}$, then $k \ll h$.

The preceding definition is motivated by the following theorem.

THEOREM 2.16. If $L$ is a characterizing sublattice of $C(X, K)$ and if $l \in L$, then $L$ is an $S$-lattice at $l$ relative to the relation $\ll_{l}$.

Proof. Suppose first that $f_{i} \ll_{l} g_{i}(i=1,2)$ in $L$. Let $k \in L$. Then there exist $g_{i}^{\prime}, h_{i} \in L$ such that $g_{i}^{\prime} \subset_{l} g_{i}, g_{i}^{\prime} \vee h_{i} \geqq k$, and $f_{i} \wedge h_{i} \leqq l$ $(i=1,2)$. Set $g^{\prime}=g_{1}^{\prime} \vee g_{2}^{\prime}$ and $h=h_{1} \wedge h_{2}$. Using (2.5) and the distributive law we have $g^{\prime} \subset_{l}\left(g_{1} \vee g_{2}\right), g^{\prime} \vee h \geqq k$, and $\left(f_{1} \vee f_{2}\right) \wedge h \leqq l$. Thus (2.11) is satisfied. Conditions (2.12), (2.13), and (2.14) are readily established by applying (1.2), (2.8), and the fact that $P(e, l)=X$ for some $e \in L$. Finally, to show that (2.15) holds, let $f, g, h, k \in L$ with $f \ll_{l} g, g \wedge k \leqq l$, and $f \vee h \in E_{l}$. Then

$$
P(f, l)^{-} \subseteq P(g, l)^{-1-1}, P(g, l)^{-1-1} \cap P(k, l)^{-}=\phi,
$$

and $P(f, l)^{-} \cup P(h, l)^{-}=X$. Therefore $P(k, l)^{-} \subseteq P(g, l)^{--{ }^{-}} \subseteq P(f, l)^{-\prime} \subseteq$ $P(h, l)^{-\prime-1}$.

3. Some fundamental properties of $S$-lattices. This section is devoted to a study of several important properties of $S$-lattices. In view of the connection between $S$-lattices and $R$-lattices (see footnote 4 ) many of the results obtained here provide sharpened versions of certain results of [13].

Throughout this section we shall assume that $L$ is an S-lattice at some element $l \in L$.

In Definition 3.7 we introduce the notion of an $S_{l}$-ideal of $L$. The

${ }^{4}$ If $L$ is a lattice that satisfies the dual of (2.11), a stronger version of (2.13), together with (2.12), (2.14), and (2.15), then it can be verified that $L$, reduced modulo a congruence ( $f$ congruent $g$ in case $f \subset_{l} g$ and $g \subset_{l} f$ ), is an $R$-lattice in the sense of Shirota $[13]$. 
main result of this section, Theorem 3.10 , states that the set of all maximal proper $S_{l}$-ideals of $L$ admits the Stone topology, relative to which it is a compact Hausdorff space.

(3.1) If $f, g \in L$ with $f \ll g$, then $f \subset_{\imath} g$.

Proof. Since $f \ll g$, there exists, by (2.14), an $h \in L$ such that $f \wedge h \leqq l$ and $g \vee h \in E_{l}$. Thus $f \subset_{l}(g \vee h)$. Since also $f \subset_{l} f$, we have by (2.5) that $f \subset_{l} f \wedge(g \vee h)=(f \wedge g) \vee(f \wedge h) \leqq(f \wedge g) \vee l$. But clearly $(f \wedge g) \vee l \subset_{l} g$; hence, by (2.2) and (2.3), it follows that $f \subset_{l} g$.

(3.2) If $f, g \in L$ with $f \ll g$, then there exist $h_{1}, h_{2} \in L$ such that $f \wedge h_{1} \leqq l, g \vee h_{2} \in E_{l}$, and $h_{2} \ll h_{1}$.

Proof. Since $f \ll g$, there exist, by (2.12), $f_{1}, f_{2} \in L$ such that $f \ll f_{1} \ll f_{2} \ll g$. Thus, by (2.14), there exist $h_{1}, h_{2} \in L$ such that $f \wedge h_{1} \leqq l$, $f_{2} \wedge h_{2} \leqq l, f_{1} \vee h_{1} \in E_{l}$, and $g \vee h_{2} \in E_{l}$. Then from $f_{2} \wedge h_{2} \leqq l, f_{1} \vee h_{1} \in E_{l}$, and $f_{1} \ll f_{2}$, together with (2.15), we have $h_{2} \ll h_{1}$.

(3.3) If $f, g, h, k \in L$ with $h \subset_{l} f, f \ll g$, and $g \subset_{l} k$, then $h \ll k$.

Proof. Let $h_{1}, h_{2} \in L$ be as in (3.2). Then $h \wedge h_{1} \leqq l$ and $k \vee h_{2} \in E_{l}$. Therefore, by (2.15), $h \ll k$.

(3.4) If $f_{i}, g_{i} \in L$ with $f_{i} \ll g_{i}(i=1,2)$, then $f_{1} \wedge f_{2} \ll g_{1} \wedge g_{2}$.

Proof. By (3.3) and (2.3) it will suffice to prove that if $f \ll g_{1}$ and $f \ll g_{2}$, then $f \ll g_{1} \wedge g_{2}$. By (3.2) there exist $h_{1}, h_{2} k_{1}, k_{2} \in L$ such that $f \wedge h_{1} \leqq l, f \wedge k_{1} \leqq l, g_{1} \vee h_{2} \in E_{l}, g_{2} \vee k_{2} \in E_{l}, h_{2} \ll h_{1}$, and $k_{2} \ll k_{1}$. Then $h_{2} \vee k_{2} \ll h_{1} \vee k_{1}$ by (2.11). Moreover, $f \wedge\left(h_{1} \vee k_{1}\right) \leqq l$ and

$$
\left(g_{1} \wedge g_{2}\right) \vee\left(h_{2} \vee k_{2}\right) \in E_{l},
$$

so that, by (2.15), $f \ll g_{1} \wedge g_{2}$.

(3.5) The set of units in $L$ for $\ll$ coincides with the set $E_{l}$ of units in $L$ for $\subset_{l}$.

Proof. By (3.1), $E_{l}$ contains every unit for $\ll$. On the other hand, by (2.13), $L$ contains a unit $e$ for $\ll$. Let $f \in E_{l}$. Then $e \subset_{l} f$, so that, from (3.3) and the fact that $e \ll e$, we have $e \ll f$. By the transitivity of $\ll$ we conclude that $f$ is a unit for $\ll$.

(3.6) For every $f \in L, l \ll f$.

Proof. Let $e \in E_{l}$ (clearly, $E_{l} \neq \phi$ ) and let $f \in L$. Then $e \wedge l \leqq l$, $e \vee f \in E_{l}$, and by (3.5), $e \ll e$. Hence by (2.15), $l \ll f$.

The remainder of this section is devoted to a study of certain ideals in $L$. We begin with the following definition.

Definition 3.7. An ideal $I$ of $L$ is an $S_{l}$-ideal in case (i) if $f \in I$, then $f \ll g$ for some $g \in I$, and (ii) if $f \in I$ and $g \ll f$, then $g \in I$. We 
denote by $\mathfrak{S}_{l}$ the set of all $S_{l}$-ideals of $L$ and by $\mathfrak{M}_{l}$ the set of all maximal proper $S_{l}$-ideals of $L$.

We note that for every $f \in L$ the set $\{g \in L ; g \ll f\}$ is an $S_{l}$-ideal. Moreover, there exists a proper $S_{l}$-ideal in $L$ provided that $f \not L l$ for some $f \in L$; in fact, by (2.4) and (3.1) this latter condition holds if and only if the ideal $\{g \in L ; g \ll l\}$ is proper.

We also observe that if $I \in \mathfrak{S}_{l}$, if $f \in I$, and if $g \subset_{l} f$, then $g \in I$. For if $f \ll h$ with $h \in I$, then $g \ll h$ by (3.3). In particular, then, $l \in I$ for every $I \in \mathfrak{S}_{l}$.

As a notational convenience we shall, for the remainder of this section, dispense with the subscripts in the symbols $\subset_{l}, E_{l}, S_{l}, \subseteq_{l}$, and $\mathfrak{M}_{l}$.

We now remark that, relative to set inclusion, $\subseteq$ is a complete lattice. In fact, if $\mathfrak{I} \subseteq \subseteq$, then, as in easily proved by (3.3), (2.11), and (2.13),

$$
\mathrm{V} \mathfrak{I}=\{f \in L ; f \ll \mathbf{V} F \text { for some finite } F \subseteq \mathbf{U} \mathfrak{I}\}
$$

and

$$
\Lambda \mathfrak{I}=\{f \in L ; f \ll g \text { for some } g \in \bigcap \mathfrak{I}\} .
$$

It is clear that if $\mathbb{S} \subseteq \mathfrak{S}$ is a chain, then $\mathrm{V} \mathfrak{S}=\mathrm{U} \mathfrak{S}$. Moreover, if $I, J \in \mathfrak{S}$, then it follows from (3.4) that $I \wedge J=I \cap J$. We therefore conclude that $\mathfrak{S}$ is an F-lattice of sets [2]. It is easy to see that a proper ideal of $L$ is s-irreducible $e^{5}$ in the lattice of ideals of $L$ if and only if it is prime. The $s$-irreducible elements of the lattice $\mathfrak{S}$ coincide with the maximal proper $S$-ideals of $L$, but need not, however, be prime ideals. ${ }^{6}$ The situation is made precise by the following lemma.

Lemma 3.8. Let $H$ be a proper S-ideal of $L$. Then the following statements are equivalent:

(i) $H \in \mathfrak{M}$.

(ii) If $f \wedge g \in H$ and $f \notin H$, then $g_{1} \in H$ for all $g_{1} \ll g$.

(iii) $H$ is s-irreducible in the lattice $\mathfrak{S}$.

Proof. (i) $\rightarrow$ (ii). Let $H \in \mathfrak{M}, f \wedge g \in H$ and $f \notin H$. Set $I=\{h \in L$; for some $k \in H$ and some $g_{1} \ll g, h \ll k \vee g_{1 j}$. It is easily verified that $I \in \mathcal{S}$ and $H \subseteq I$; thus, by the maximality of $H$, either $H=I$ or $L=I$. If the latter holds, then $f \in I$, so that there exist $k \in H$ and $g_{1} \ll g$ such that $f \ll k \vee g_{1}$, and thus $f \subset\left(k \vee g_{1}\right)$. But then $f \subset\left(k \vee g_{1}\right) \wedge f \subset(k \vee g) \wedge f=$ $(k \wedge f) \vee(g \wedge f)$. However, $k \wedge f$ and $g \wedge f$ are elements of $H$, so

${ }^{5}$ An element $x$ of a lattice $L$ is $s$-irreducible [2] in case (i) $x \geqq y$ for some $y \in L$, and (ii) if $a, b \in L$ with $a \wedge b \leqq x$, then either $a \leqq x$ or $b \leqq x$.

6 Since $X$ is compact Hausdorff, it can be shown that if $l \in C(X, R)$, then every $s$-irreducible $S_{l}$-ideal of $C(X, R)$ is prime if and only if $X$ is discrete. 
that $f \in H$, contrary to hypothesis. Hence $I=H$, and since it is evident by (2.12) that $g_{1} \in I$ for all $g_{1} \ll g$, $H$ satisfies (ii).

(ii) $\rightarrow$ (iii). Since $H$ is proper, we need only prove that if $I, J \in \mathfrak{S}$ and if $I \wedge J \subseteq H$, then either $I \subseteq H$ or $J \subseteq H$. If $I \nsubseteq H$, then $g_{1} \notin H$ for some $g_{1} \in I$. Let $g \in I$ be such that $g_{1} \ll g$ and let $f$ be any element of $J$. Since $f \wedge g \in I \cap J$ and $I \cap J=I \wedge J$, it follows that $f \wedge g \in H$. Hence $f \in H$.

(iii) $\rightarrow$ (i). Since $E \neq \phi$, Zorn's lemma will supply an $M \in \mathfrak{M}$ such that $H \subseteq M$. If $H \neq M$, then there exist $f \ll f_{1} \ll f_{2}$ with $f \notin H$ and $f_{2} \in M$. By (3.2) there exist $h_{2} \ll h_{1}$ such that $f_{1} \wedge h_{1} \leqq l$ and $f_{2} \vee h_{2} \in E$. Since $l \in H$, we must have $f_{1} \wedge h_{1} \in H$. Let $I\left(f_{1}\right)=\left\{k \in L ; k \ll f_{1}\right\}$ and $I\left(h_{1}\right)=\left\{k \in L ; k \ll h_{1}\right\}$. Then $I\left(f_{1}\right), I\left(h_{1}\right) \in \mathfrak{S}$, and, by (3.4), $I\left(f_{1}\right) \cap I\left(h_{1}\right) \subseteq$ $H$. Thus, by the $s$-irreducibility of $H$, and since $f \notin H$, it is clear that $h_{2} \in H$. Therefore $h_{2} \in M$, so that $f_{2} \vee h_{2} \in M \cap E$, a contradiction. Hence $H=M$, as desired.

In view of the preceding lemma and Theorems 1.1 and 1.2 of [2], the set $M$ admits the Stone topology relative to which it is $T_{1}$. Moreover, since $E \neq \phi$, Theorem 3.4 of [2] implies that $\mathfrak{M}$ is compact in this topology. Throughout the remainder of this paper whenever $\mathfrak{M}$ is considered as a topological space, its topology will be this "Stone topology".

Lemma 3.9. For each $f \in L$, the set $\mathfrak{F}(f)=\{M \in \mathfrak{M} ; f \notin M\}$ is closed in $\mathfrak{M}$.

Proof. Recall first that if $\mathfrak{A} \subseteq \mathfrak{M}$ and if $\mathfrak{T}^{*}=\Lambda\{M \in \mathfrak{M} ; M \in \mathfrak{A}\}$, then $\mathfrak{A}^{-}=\left\{M \in \mathfrak{M} ; \mathfrak{P}^{*} \subseteq M\right\}$. Now let $f \in L, M \in \mathfrak{M}$, and $\mathfrak{F}(f)^{*} \subseteq M$; we must prove that $f \notin M$. Suppose on the contrary that $f \in M$. Then $f \ll g$ for some $g \in M$. By (3.2) and (2.12) there exist $h_{2} \ll k \ll h_{1}$ such that $f \wedge h_{1} \leqq l$ and $g \vee h_{2} \in E$. If $N \in \mathfrak{F}(f)$, then $f \wedge h_{1} \in N$ so that, by Lemma $3.8, k \in N$. This implies that $h_{2} \in \mathfrak{F}(f)^{*}$, and therefore $g \vee h_{2} \in M \cap E$, a contradiction. Hence $f \notin M$, and the proof is complete.

We can now prove the main result of this section ( $f$. [13, Theorem 2]).

TheOREM 3.10. Let $L$ be an S-lattice at the element $l \in L$. Then the set $\mathfrak{M}_{l}$ of all maximal $S_{l}$-ideals admits the Stone topology, and, relative to this topology, $\mathfrak{M}_{l}$ is a compact Hausdorff space.

Proof. From the preceding remarks it will suffice to prove that $\mathfrak{M}_{l}$ is Hausdorff. Let $M \neq N$ in $\mathfrak{M}_{l}$ so that there exist $f \notin M$ and $g \in N$ with $f \ll g$. By (3.2) and Lemma 3.8, there exists an element $h \in L$ such that $h \in M$ and $h \vee g \in E_{l}$. Thus $M \notin \mathfrak{F}(h), N \notin \mathfrak{F}(g)$, and $\mathfrak{F}(h) \cup \mathfrak{F}(g)=\mathfrak{M}_{l}$.

4. Maximal $S_{l}$-ideals in characterizing sublattices of $C(X, K)$. Let $L$ be a characterizing sublattice of $C(X, K)$ and let $l \in L$. We first 
obtain a characterization of the maximal $S_{l}$-ideals of $L$. This characterization is then used to show that $L$ determines the topology of $X$.

If $L$ is a characterizing sublattice of $C(X, K)$, and if $l \in L$, then for each $x \in X$ set

$$
M_{l}(x)=\left\{f \in L ; x \in P(f, l)^{-\prime}\right\} .
$$

It is evident that each $M_{l}(x)$ is an ideal, and in fact, by (2.8) and (1.2), an $S_{l}$-ideal in $L$. Since it is clear that if $x \neq y$ in $X$, then $M_{l}(x) \neq$ $M_{l}(y)$, we have proved a portion of the following key result:

THEOREM 4.1. If $L$ is a characterizing sublattice of $C(X, K)$, and if $l \in L$, then a subset $M$ of $L$ is a maximal proper $S_{l}$-ideal of $L$ if and only if there exists a (necessarily unique) element $x \in X$ such that $M=M_{l}(x)$.

Proof. In view of the above remarks it will suffice to prove that each $M_{l}(x)$ is maximal in $\mathfrak{S}_{l}$ and that each $M \in \mathfrak{M}_{l}$ is contained in some $M_{l}(x)$. Suppose first that $f \wedge g \in M_{l}(x), f \notin M_{l}(x)$, and $g^{\prime} \ll_{l} g$. Then $x \in P(f, l)^{-}$. Thus if $g^{\prime} \notin M_{l}(x)$, then $x \in P\left(g^{\prime}, l\right)^{-} \subseteq P(g, l)^{-1-1}$, and hence it follows that $x \in\left[P(g, l)^{-1-1} \cap P(f, l)\right]^{-}=[P(g, l) \cap P(f, l)]^{-}=$ $P(f \wedge g, l)^{-}$, contrary to $f \wedge g \in M_{l}(x)$. Therefore, by Lemma 3.8, $M_{l}(x) \in \mathfrak{M}_{l}$.

Conversely, let $M \in \mathfrak{M}_{l}$ and suppose that for each $x \in X$ there is an $f_{x} \in M$ with $x \in P\left(f_{x}, l\right)^{-}$. Then for each $x$ there is an $h_{x} \in M$ such that $f_{x} \ll_{l} h_{x}$, and hence $x \in P\left(h_{x}, l\right)^{-\prime-1}$. Now since $X$ is compact, there is a finite set $x_{1}, \cdots, x_{n} \in X$ such that

$$
X=\bigcup_{i=1}^{n} P\left(h_{x_{i}}, l\right)^{-\prime-\prime} \subseteq P\left(\bigvee_{i=1}^{n} h_{x_{i}}, l\right)^{-} .
$$

But then

$$
g \subset_{\imath} \bigvee_{i=1}^{n} h_{x_{i}}
$$

for every $g \in L$, and

$$
\bigvee_{i=1}^{n} h_{x_{i}} \in M
$$

contrary to $g \notin M$ for some $g \in L$. Thus $M \subseteq M_{l}(x)$ for some $x \in X$.

DEFINITION 4.2. If $L$ is a characterizing sublattice of $C(X, K)$, if $l \in L$, and if $x \in X$, then we say that the maximal $S_{l}$-ideal $M_{l}(x)$ is associated with $x$.

The preceding theorem then asserts that each maximal $S_{l}$-ideal of $L$ is associated with a unique point in $X$. 
THEOREM 4.3. If $L$ is a characterizing sublattice of $C(X, K)$, and if $l \in L$, then the mapping $M_{l}(x) \rightarrow x$ is a homeomorphism of the space $\mathfrak{M}_{2}$ onto $X$. Thus the space $X$ is determined by any characterizing sublattice of $C(X, K)$.

Proof. By Theorem 4.1 the mapping $M_{l}(x) \rightarrow x$ is one-to-one from $\mathfrak{M}_{l}$ onto $X$; since $\mathfrak{M}_{l}$ is compact, it will therefore suffice to prove that this mapping is continuous. Thus let $U$ be a neighborhood of $x \in X$ and let $f \in L$ be such that $f<l$ at $x$ and $f>l$ on $U^{\prime}$. By Lemma 3.9, the set

$$
\mathfrak{U}=\left\{M \in \mathfrak{M}_{l} ; f \in M\right\}
$$

is open in $\mathfrak{M}_{l}$, and clearly $M_{l}(x) \in \mathfrak{U}$. To complete the proof we show that $y \in U$ for every $M_{l}(y) \in \mathfrak{U}$. If $M_{l}(y) \in \mathfrak{U}$, then $y \in P(f, l)^{-1}$; hence $f(y) \leqq l(y)$. But $f>l$ on $U^{\prime}$ so that $y \in U$, as desired.

5. C-lattices. Motivated by the notion of a characterizing $Q$-sublattice of $C(X, K)$ we introduce in this section a class of abstract lattices, called $C$-lattices, and an important subclass, called $C_{1}$-lattices. The main result of this section is that every characterizing $Q$-sublattice of $C(X, K)$ is a $C_{1}$-lattice.

Definition 5.1. If $L$ is a distributive lattice and if $\alpha, \beta \in L$, then we write $\alpha-3 \beta$ in case $\alpha<\beta$ and, for every $f, g \in L$, the following conditions hold:

(i) If $f \vee g \geqq \beta$, and if $g \leqq \alpha$, then $f \geqq \beta$.

(ii) If $f \wedge g \leqq \alpha$, and if $g \geqq \beta$, then $f \leqq \alpha$.

It is readily seen that 3 is a transitive relation on $L$ Moreover, if $\gamma \leqq \alpha$, if $\alpha-3 \beta$, and if $\beta \leqq \delta$, then $\gamma-3 \delta$.

(5.2) If $L$ is a distributive lattice, and if $\alpha-3 \beta$ in $L$, then $\beta$ is a unit for $\subset_{\alpha}$ in $L$.

Proof. Let $\alpha 3 \beta$ in $L$ and let $g \in L$. If $f \in L$ with $f \wedge \beta \leqq \alpha$, then, since $\beta \geqq \beta$, we have $f \leqq \alpha$, so that $f \wedge g \leqq \alpha$. That is, $g \subset_{\alpha} \beta$.

(5.3) If $L$ is a distributive lattice, if $\alpha-3 \beta$ in $L$, and if $f_{i}, g_{i} \in L$ $(i=1,2)$, then

(i) $f_{1} \vee g_{1} \geqq \beta, f_{2} \vee g_{2} \geqq \beta$, and $f_{1} \wedge f_{2} \leqq \alpha$ imply $g_{1} \vee g_{2} \geqq \beta$;

(ii) $f_{1} \wedge g_{1} \leqq \alpha, f_{2} \wedge g_{2} \leqq \alpha$, and $f_{1} \vee f_{2} \geqq \beta$ imply $g_{1} \wedge g_{2} \leqq \alpha$.

Proof. If $f_{i} \vee g_{i} \geqq \beta(i=1,2)$, then using the distributive law we have $\left(f_{1} \wedge f_{2}\right) \vee\left(g_{1} \vee g_{2}\right) \geqq \beta$. Thus if $\alpha-3 \beta$, and if $f_{1} \wedge f_{2} \leqq \alpha$, we have $g_{1} \vee g_{2} \geqq \beta$, establishing (i). In a similar manner (ii) is proved. 
Definition 5.4. A non-empty subset $Q$ of a distributive lattice $L$ is a separating chain in $L$ in case, relative to $-3, Q$ is a chain having neither a first nor a last element.

(5.5) If $L$ is a $Q$-sublattice of $C(X, K)$, then $Q$ is a separating chain in $L$.

Proof. It will suffice to prove that if $\alpha, \beta \in Q$, and if $\alpha<\beta$, then $\alpha-3 \beta$. Thus suppose that $f, g \in L$ with $g \leqq \alpha$ and $f \geqq \beta$. Then $f(x)<\beta$ for some $x \in X$ and, since $g(x) \leqq \alpha$, it follows that $(f \vee g)(x) \leqq f(x) \vee \alpha<\beta$. Similarly, if $g \geqq \beta$ and $f \geqq \alpha$, then $f \wedge g \not \alpha$. Hence $\alpha-\beta$.

Lemma 5.6. Let $L$ be a characterizing Q-sublattice of $C(X, K)$, let $\alpha, \beta, \gamma, \delta \in Q$, and let $f \in C(X, K)$. If $\beta<\gamma$, then there exist $k, l, m \in L$ such that $k \vee m \geqq \delta, f \wedge m \leqq \gamma, k \wedge l \leqq \alpha$, and $f \vee l \geqq \beta$.

Proof. Since $Q$ is dense-in-itself, there exist $\rho, \eta \in Q$ such that $\beta<\rho<\eta<\gamma$. Now the closed sets $P(f, \eta)^{-}, P(f, \gamma)^{-}$, and $P(f, \rho)^{-}$ are disjoint, respectively, from the closed sets $P(f, \rho)^{\prime}, P(f, \eta)^{\prime}$, and $P(f, \beta)^{\prime}$. Therefore since $L$ is characterizing, there exist, by (1.2), elements $k, l, m \in L$ such that $P(f, \eta)^{-} \subseteq P(k, \delta), P(f, \rho)^{\prime} \subseteq P(k, \alpha)^{\prime}$, $P(f, \gamma)^{-} \subseteq P(m, \gamma)^{\prime}, P(f, \eta)^{\prime} \subseteq P(m, \delta), P(f, \rho)^{-} \subseteq P(l, \alpha)^{\prime}$, and $P(f, \beta)^{\prime} \subseteq$ $P(l, \beta)$. Since $X=P(f, \eta) \cup P(f, \eta)^{\prime} \subseteq P(k, \delta) \cup P(m, \delta)=P(k \vee m, \delta)$, we have $k \vee m \geqq \delta$, and since $P(f, \gamma) \subseteq P(m, \gamma)^{\prime}$, we have $f \wedge m \leqq \gamma$. In a similar fashion it follows that $k \wedge l \leqq \alpha$ and $f \vee l \geqq \beta$.

Motivated by the preceding lemma we introduce the following definition:

Definition 5.7. It $L$ is a distributive lattice and if $Q$ is a separating chain in $L$, then the "stretching function" $\mathscr{S}_{Q}{ }^{L}$ is the function on the cartesian product $L \times Q^{4}$ to the subsets of the cartesian product $L^{3}$ defined as follows: $(k, l, m) \in \mathscr{S}_{Q}^{L}(f, \alpha, \beta, \gamma, \delta)$ in case

$$
\begin{array}{ll}
k \vee m \geqq \delta, & f \wedge m \leqq \gamma, \\
k \wedge l \leqq \alpha, & f \vee l \geqq \beta .
\end{array}
$$

Whenever no confusion is likely, we shall omit the indices $L$ and $Q$ in the symbol $\mathscr{S}_{Q}{ }^{L}$.

We observe that in most cases $\mathscr{S}(f, \alpha, \beta, \gamma, \delta)=\phi$. However it is easily seen that if $\beta \leqq \alpha$, if $\delta \leqq \gamma$, or if $f \leqq \gamma$, then $\mathscr{S}(f, \alpha, \beta, \gamma, \delta) \neq \phi$.

Definition 5.8. Let $L$ be a distributive lattice, let $Q$ be a nonempty subset of $L$, and consider the following conditions:

(C.0) $Q$ is a separating chain in $L$.

(C.1) If $\beta, \gamma \in Q$ with $\beta<\gamma$, then $\mathscr{S}_{Q}^{L}(f, \alpha, \beta, \gamma, \delta) \neq \phi$ for all 
$\alpha, \delta \in Q$ and all $f \in L$.

(C.2) $Q$ is dense-in-itself.

(C.2) If $f \in L$, if $\alpha \in Q$, and if $f \leqq \beta$ for all $\beta>\alpha$ in $Q$, then $f \leqq \alpha$.

(C.3) If $f, g \in L$ and if $f \subset_{\alpha} g$ for all $\alpha \in Q$, then $f \leqq g$. We say that $L$ is a $C$-lattice relative to $Q$ in case (C.0), (C.1), and (C.2) are satisfied. We say that $L$ is a $C_{1}$-lattice relative to $Q$ in case (C.0), (C.1), (C.2'), and (C.3) are satisfied.

We observe that (C.0) and (C.2') together imply (C.2). For if $\alpha<\beta$ in $Q$, then there is a $\gamma \in Q$ with $\gamma>\alpha$ and $\beta \not \Varangle \gamma$; that is, $\alpha<\gamma<\beta$. Thus every $C_{1}$-lattice is also a $C$-lattice.

We remark also that the defining conditions for a $C$-lattice and for a $C_{1}$-lattice are all arithmetical relative to the system $\left\langle L, \mathrm{~V}, \Lambda, \mathscr{E}_{Q}\right\rangle$. That is, each is formulated solely in terms of (i) elements of $L$, (ii) elementary logical constants (connectives, quantifiers, identity symbol), (iii) the operations $V$ and $\Lambda$, and (iv) the predicate $\mathscr{E}_{Q}$ expressing elementhood in $Q$ ( $c f$. [17] and the Introduction).

The following examples show that, in the presence of (C.0), conditions (C.1), (C.2'), and (C.3) are independent, and that (C.2') is not implied by (C.1), (C.2), and (C.3).

EXAMPLE 5.9. Let $R(e)$ be the chain obtained by adjoining to the real chain $R$ an element $e \notin R$ in such a manner that $0<e<\alpha$ for every $\alpha>0$ in $R$. If $Q=\{\alpha \in R ; \alpha \neq 0\}$, then $Q$ is a separating chain in $R(e)$, and, relative to $Q, R(e)$ satisfies (C.1) and (C.2'). However $R(e)$ is. not a $C_{1}$-lattice relative to $Q$ since $e \subset_{\alpha} 0$ for all $\alpha \in Q$.

EXAMPLE 5.10. The chain $R$ is also a separating chain in $R(e)$, but, relative to $R, R(e)$ is not a $C_{1}$-lattice since $e \not 0$ and yet $e<\alpha$ for all $\alpha>0$ in $R$. We note, however, that, relative to $R, R(e)$ satisfies (C.1), (C.2), and (C.3).

ExAMPLE 5.11. Let $L$ be the set of all real-valued functions $f$ on the two element set $\{x, y\}$ such that $|f(x)-f(y)| \leqq 1$. Then $L$ is a distributive lattice, the set $R$ of constants functions in $L$ is a separating chain, and, relative to $R, L$ satisfies (C.2) and (C.3). However, $L$ fails to satisfy (C.1), and hence it is not a $C$-lattice.

We now prove the main result of this section.

THEOREM 5.12. If $L$ is a characterizing Q-sublattice of $C(X, K)$, then $L$ is a $C_{1}$-lattice relative to $Q$.

Proof. By (5.5), $Q$ is a separating chain in $L$, and, since $Q$ is order-dense in $K$, it is clear that $\left(\mathrm{C} .2^{\prime}\right)$ is satisfied. Moreover, Lemma 
5.6, shows that $L$ satisfies (C.1). Finally, to prove that $L$ satisfies (C.3), suppose that $f \not Z g$ in $L$. Then for some $x \in X$ and some $\alpha \in Q, f(x)>\alpha>g(x)$. Thus, by the continuity of $g$, we have $P(f, \alpha)^{-} \nsubseteq$ $P(g, \alpha)^{-}$so that, by (2.8), $f \not \subset_{\alpha} g$.

6. Some fundamental properties of $C$-lattices. Of the two major results of this section the first, an analogue of Theorem 2.16 , states that if $L$ is a $C$-lattice relative to the separating chain $Q$ in $L$, then for each $\alpha \in Q$ there is a transitive binary relation $\leqslant_{a}$ on $L$ relative to which $L$ is an $S$-lattice at $\alpha$. Thus each $\alpha \in Q$ determines a lattice $\mathfrak{S}_{\alpha}$ of $S_{\alpha^{-}}$ ideals and its associated compact Hausdorff space $\mathfrak{M}_{\alpha}$. The second major result establishes a certain homogeneity in $L$. Explicity, if $L$ is as above, then for every pair $\alpha, \beta \in Q$ there is an isomorphism $\Phi$ of $\mathfrak{S}_{\alpha}$ onto $\mathfrak{S}_{\beta}$, and consequently there is a homeomorphism of $\mathfrak{M}_{\alpha}$ onto $\mathfrak{M}_{\beta}$. Moreover, $\Phi$ has the property that if $\alpha \leqq \beta$, then $I \subseteq \Phi(I)$ for all $I \in \mathfrak{S}_{\alpha}$.

Throughout this section we shall assume that $L$ is a C-lattice relative to the separating chain $Q .^{7}$

We shall adopt the convention that all lower case letters, Greek and Latin, will denote elements of $L$. In particular, lower case Greek letters will be reserved for elements in the chain $Q$.

(6.1) If $\gamma<\eta \leqq \delta$, if $f \vee h \geqq \eta$, and if $(k, l, m) \in \mathscr{S}(f, \alpha, \beta, \gamma, \delta)$, then $k \vee h \geqq \eta$.

Proof. In view of $f \vee h \geqq \eta, k \vee m \geqq \delta$, and $f \wedge m \leqq \gamma$, the desired inequality follows from (5.3).

Definition 6.2. For each $\alpha \in Q$ we define the relations $\prec_{\infty}$ and on $L$ as follows:

(i) $f \prec_{\alpha} g$ in case $\mathrm{g} \vee h \geqq \beta$ and $f \wedge h \leqq \alpha$ for some $h \in L$ and some $\beta>\alpha$ in $Q$.

(ii) $f \ll_{\alpha} g$ in case $f \prec_{\alpha} g^{\prime}$ for some $g^{\prime} \subset_{\alpha} g .^{8}$

It is clear that if $f \prec_{\alpha} g$ in $L$, then $f \ll_{\alpha} g$.

(6.3) If $f \ll_{\alpha} g$ in $L$, then $f \subset_{\alpha} g$.

Proof. Since by (2.2) the relation $\subset_{\alpha}$ is transitive, it will suffice to prove that $f \prec_{\alpha} g$ implies $f \subset_{\alpha} g$. But if $g \vee h \geqq \beta, f \wedge h \leqq \alpha$, and $\beta>\alpha$, then, by (5.3), $k \wedge g \leqq \alpha$ implies $k \wedge f \leqq \alpha$. That is, $f \subset_{\alpha} g$.

(6.4) Both $\prec_{\alpha}$ and $\ll_{\infty}$ are transitive relations on $L$.

Proof. This follows from (6.3) and the obvious fact that if $f_{1} \subset_{\alpha} f$

7 Propositions (6.1) and (6.3)-(6.6) actually require only that $L$ be a distributive lattice and that $Q$ be a separating chain in $L$.

${ }^{8}$ If $L$ is a characterizing $Q$-sublattice of $C(X, K)$ and if $\alpha \in Q$, then it is easily seen that the definitions of $\ll_{\alpha}$ in 6.2 and 2.6 coincide. 
and $f \prec_{x} g$, then $f_{1} \prec_{x} g$.

(6.5) If $\alpha<\beta$, if $\gamma<\delta$, and if $(k, l, m) \in \mathscr{S}(f, \alpha, \beta, \gamma, \delta)$, then $f \prec_{\gamma} k$ and $k \prec_{\alpha} f$.

Proof. Since $(k, l, m) \in \mathscr{S}(f, \alpha, \beta, \gamma, \delta)$, we have $f \wedge m \leqq \gamma$, and $k \vee m \geqq \delta$; hence $f \prec_{\gamma} k$. Also, $k \wedge l \leqq \alpha$ and $f \vee l \geqq \beta$ together imply that $k \prec_{\alpha} f$.

(6.6) Let $\alpha<\beta$ and $\gamma<\delta$. If $(k, l, m) \in \mathscr{P}(f, \alpha, \beta, \gamma, \delta)$, and if $\left(k^{\prime}, l^{\prime}, m^{\prime}\right) \in \mathscr{S}(k, \alpha, \beta, \gamma, \delta)$, then $k \prec_{\gamma} k^{\prime}, k^{\prime} \prec_{\alpha} k, m^{\prime} \prec_{\gamma} m$, and $l \prec_{\alpha} l^{\prime}$.

Proof. The first two relations follow from (6.5). From $k \vee m \geqq \delta$ and $k \wedge m^{\prime} \leqq \gamma$ we have $m^{\prime} \prec_{\gamma} m$, and from $k \vee l^{\prime} \geqq \beta$ and $k \wedge l \leqq \alpha$, it follows that $l \prec_{a} l^{\prime}$.

(6.7) If $L$ is a C-lattice relative to the separating chain $Q$, and if $\alpha \in Q$, then $L$ satisfies (2.11), (2.12), (2.13), and (2.14) at $\alpha$ relative to $\prec_{\alpha}$.

Proof. For $i=1,2$, let $f_{i} \prec_{\alpha} g_{i}$ so that for some $h_{i} \in L$ and some $\beta_{i} \in Q$ we have $g_{i} \vee h_{i} \geqq \beta_{i}, f_{i} \wedge h_{i} \leqq \alpha$, and $\beta=\beta_{1} \wedge \beta_{2}>\alpha$. Then

$$
\begin{aligned}
\left(g_{1} \vee \vee g_{2}\right) \vee\left(h_{1} \wedge h_{2}\right) & =\left(g_{1} \vee g_{2} \vee h_{1}\right) \wedge\left(g_{1} \vee g_{2} \vee h_{2}\right) \\
& \geqq\left(g_{1} \vee h_{1}\right) \wedge\left(g_{2} \vee h_{2}\right) \\
& \geqq \beta>\alpha,
\end{aligned}
$$

and similarly,

$$
\left(f_{1} \vee f_{2}\right) \wedge\left(h_{1} \wedge h_{2}\right) \leqq \alpha
$$

Thus $f_{1} \vee f_{2} \prec_{\alpha} g_{1} \vee g_{2}$ and $\prec_{\alpha}$ satisfies (2.11). Now let $f \prec_{\alpha} g$ and let $h \in L, \delta \in Q$ be such that $f \wedge h \leqq \alpha$ and $g \vee h \geqq \delta>\alpha$. Since $Q$ is dense-in-itself, there exist $\beta, \gamma \in Q$ with $\alpha<\beta<\gamma<\delta$, and since $L$ satisfies (C.1) relative to $Q$, there exists $(k, l, m) \in \mathscr{S}(g, \alpha, \beta, \gamma, \delta)$. By (6.5) we have $k \prec_{\alpha} g$, and by (6.1) we have $k \vee h \geqq \delta$, so that $f \prec_{\alpha} k$. Hence $f \prec_{\alpha} k \prec_{\alpha} g$, and (2.12) holds. If $\beta>\alpha$, then $f \prec_{\alpha} \beta$ for all $f \in L$; hence $\beta$ is a unit for $\prec_{\alpha}$ and (2.13) is satisfied. Suppose, finally, that $f \prec_{\alpha} g$ so that $g \vee h \geqq \beta>\alpha$ and $f \wedge h \leqq \alpha$ for some $h \in L$ and some $\beta \in Q$. By (5.2), $\beta \in E_{\alpha}$; hence $g \vee h \in E_{\alpha}$ and (2.14) is established.

THEOREM 6.8. If $L$ is a C-lattice relative to a separating chain $Q$, and if $\alpha \in Q$, then $L$ is an $S$-lattice at $\alpha$ relative to the relation $<_{\alpha}$.

Proof. That $L$ satisfies (2.11), (2.12), (2.13), and (2.14) relative to $\ll_{x}$ follows readily from (6.7). For example, if $f_{i} \ll_{x} g_{i}$, then there exist $g_{i}^{\prime} \in L$ such that $f_{i} \prec_{\alpha} g_{i}^{\prime}$ and $g_{i}^{\prime} \subset_{\alpha} g_{i}(i=1,2)$. Then by (6.7) and 
(2.5) we have $f_{1} \vee f_{2} \prec_{\alpha} g_{1}^{\prime} \vee g_{2}^{\prime}$ and $g_{1}^{\prime} \vee g_{2}^{\prime} \subset_{\alpha} g_{1} \vee g_{2}$; hence $f_{1} \vee f_{2} \ll_{\alpha} g_{1} \vee g_{2}$. Finally, to establish (2.15) suppose that $f \ll_{\alpha} g, g \wedge k \leqq \alpha$, and $f \vee h \in E_{\alpha}$. Then for some $g_{1}, h_{1} \in L$ and some $\beta>\alpha$ in $Q, g_{1} \supset_{\alpha} g, h_{1} \wedge f \leqq \alpha$, and $h_{1} \vee g_{1} \geqq \beta$. Since $g_{1} \subset_{\alpha} g$ and $g \wedge k \leqq \alpha$, it follows that $g_{1} \wedge k \leqq \alpha$. But this, together with $h_{1} \vee g_{1} \geqq \beta$, implies $k \prec_{\alpha} h_{1}$. Thus in order to prove the desired relation, $k \ll_{\alpha} h$, it will suffice to show that $h_{1} \subset_{\alpha} h$. Therefore let $l \in L$ with $l \wedge h \leqq \alpha$. Then $\left(l \wedge h_{1}\right) \wedge(f \vee h) \leqq \alpha$. However, since $f \vee h \in E$, we have $\left(l \wedge h_{1}\right) \wedge \beta \leqq \alpha$ for every $\beta>\alpha$. Therefore, since $Q$ is a separating chain, $l \wedge h_{1} \leqq \alpha$. That is, $h_{1} \subset_{\alpha} h$, as desired.

From Theorems 3.10 and 6.8 we conclude that if $L$ is a $C$-lattice relative to the separating chain $Q$, then there is associated with each $\alpha \in Q$ a compact Hausdorff space, namely, the space $\mathfrak{M}_{r}$ of maximal $S_{x^{-}}$ ideals of $L$. We conclude this section by proving that these spaces are pairwise homeomorphic.

DeFinition 6.9. If $\alpha \leqq \beta$, then we define the mappings $\Phi_{\alpha \beta}: \mathfrak{S}_{\alpha} \rightarrow \mathfrak{S}_{\beta}$ and $\Phi^{\alpha_{\beta}}: \mathfrak{S}_{\beta} \rightarrow \mathfrak{S}_{\alpha}$ as follows: For each $I \in \mathfrak{S}_{\alpha}$ and each $J \in \mathfrak{S}_{\beta}$,

$$
\Phi_{\alpha \beta} I=\Lambda\left\{H \in \mathfrak{S}_{\beta} ; I \subseteq H\right\},
$$

and

$$
\Phi^{\alpha \beta} J=\mathrm{V}\left\{H \in \mathfrak{S}_{\alpha} ; H \subseteq J\right\} .
$$

Since $\mathfrak{S}_{\alpha}$ and $\mathfrak{S}_{\beta}$ are complete lattices, it is clear that $\Phi_{\alpha \beta}$ and $\Phi^{\alpha \beta}$ are single-valued mappings. We intend to prove that $\Phi_{\alpha \beta}$ is, in fact, an isomorphism of $\mathfrak{S}_{\alpha}$ onto $\mathfrak{S}_{\beta}$ and that $\Phi^{\alpha \beta}$ is its inverse. This is obviously the case for $\alpha=\beta$.

$$
\begin{aligned}
& \text { If } \alpha<\beta \text { and if } I \in \mathfrak{S}_{\alpha} \text {, set } \\
& I^{*}=\left\{k \in L ; k \prec_{\alpha} g \text { and } g \prec_{\beta} k \text { for some } g \in I\right\} .
\end{aligned}
$$

Then

$$
\Phi_{\alpha \beta} I=\left\{f \in L ; f \prec_{\beta} k \text { for some } k \in I^{*}\right\} \text {, }
$$

and

$$
I^{*} \subseteq I \subseteq \Phi_{\alpha \beta} I
$$

Proof. First set $I^{\wedge}=\left\{f \in L ; f \prec_{\beta} k\right.$ for some $\left.k \in I^{*}\right\}$. Since $k \in I^{*}$ implies that $k \ll_{\alpha} g$ for some $g \in I$, it follows that $k \in I$; hence $I^{*} \subseteq I$. Next it is easily seen, using (6.7), that $I^{*}$ is closed under joins. Hence, again from (6.7), $I^{\wedge}$ is an ideal in $L$; in fact, $I^{\wedge} \in \widetilde{S}_{\beta}$. Now let $f \in I$ so that for some $g \in I$ both $f \leqq g$ and $f \prec_{\alpha} g$. Since $L$ is a $C$-lattice relative to $Q$, it follows from (6.5) that there is a $k \in L$ with $k \prec_{\alpha} g$ and $g \prec_{\beta} k$. 
Thus $k \in I^{*}$. But $f \leqq g$ then implies $f \prec_{\beta} k$; hence $f \in I^{\wedge}$. Since $f$ is arbitrary in $I$, this implies the inclusion $I \subseteq I^{\wedge}$. From this and the fact that $I^{\wedge} \in \mathfrak{S}_{\beta}$ we also conclude $\Phi_{\alpha \beta} I \subseteq I^{\wedge}$. On the other hand, let $J \in \mathfrak{S}_{\beta}$ with $I \subseteq J$; then $I^{*} \subseteq J$. If $f \in I^{\wedge}$, then $f \prec_{\beta} k$ for some $k \in I^{*}$; hence $f \in J$. That is, $I^{\wedge} \subseteq J$, so that $I^{\wedge} \subseteq \Phi_{\alpha \beta} I$. Thus the proof is complete.

(6.11) If $\alpha<\beta$ and if $f \prec_{\alpha} \bigvee_{i=1}^{n} g_{i}$, then there exist $k_{i} \in L(i=$ $1, \cdots, n)$ such that

(i) $k_{i} \prec_{\alpha} g_{i} \quad(i=1, \cdots, n)$;

(ii) $f \prec_{\beta} \bigvee_{i=1}^{n} k_{i}$;

(iii) if $\bigvee_{i=1}^{n} k_{i} \prec_{\beta} k^{\prime}$, then $f \prec_{\alpha} k^{\prime}$.

Proof. Since $f \prec_{\alpha} \bigvee_{i=1}^{n} g_{i}$, there is an $h \in L$ such that $f \wedge h \leqq \alpha$ and $h \vee\left(\bigvee_{i=1}^{n} g_{i}\right) \geqq \zeta>\alpha$. Let $\alpha<\alpha_{1}<\alpha_{2}<\zeta \wedge \beta \leqq \zeta \vee \beta<\beta_{1}$ and, for each $i=1, \cdots, n$, $\operatorname{let}\left(k_{i}, l_{i}, m_{i}\right) \in \mathscr{S}\left(g_{i}, \alpha, \alpha_{1}, \alpha_{2}, \beta_{1}\right)$ so that, by (6.5), $k_{i} \prec_{\alpha} g_{i}$. Set $g=\bigvee_{i=1}^{n} g_{i}, k=\bigvee_{i=1}^{n} k_{i}, m=\bigwedge_{i=1}^{n} m_{i}$, and $l=\bigwedge_{i=1}^{n} l_{i}$. Then, using the distributive law, it is easily proved that $(k, l, m) \in \mathscr{S}\left(g, \alpha, \alpha_{1}, \alpha_{2}, \beta_{1}\right)$. Thus from $g \wedge m \leqq \alpha_{2}, f \wedge h \leqq \alpha<\alpha_{2}, g \vee h \geqq \zeta>\alpha_{2}$, and (5.3) we conclude that $f \wedge m \leqq \alpha_{2}<\beta$. But then since $k \vee m \geqq \beta_{1}>\beta$, we have $f \prec_{\beta} k$. Finally, suppose that $k \prec_{\beta} k^{\prime}$, so that $k \wedge h^{\prime} \leqq \beta$ and $k^{\prime} \vee h^{\prime} \geqq$ $\eta>\beta$ for some $h^{\prime} \in L$ and some $\eta \in Q$. Combining these two inequalities with $k \vee m \geqq \beta_{1}>\beta$ and using (5.3) we have $k^{\prime} \vee m \geqq \eta \wedge \beta_{1}>\beta$. But $k^{\prime} \vee m>\beta, g \vee h \geqq \zeta, g \wedge m \leqq \alpha_{2}<\zeta \wedge \beta$, and (5.3) imply that $k^{\prime} \vee h \geqq \zeta \wedge \beta>\alpha$. Therefore $f \prec_{\alpha} k^{\prime}$ as desired.

(6.12) If $\alpha<\beta$, and if $I, J \in \mathfrak{S}_{\alpha}$, then $I \subseteq J$ if and only if $\Phi_{\alpha \beta} I \subseteq \Phi_{\alpha \beta} J$.

Proof. Clearly $I \subseteq J$ implies $\Phi_{\alpha \beta} I \subseteq \Phi_{\alpha \beta} J$. Thus suppose that $\Phi_{\alpha \beta} I \subseteq \Phi_{\alpha \beta} J$ and let $f \in I$. For some $g \in I$ with $f \prec_{\alpha} g$, let $k \in L$ satisfy (i), (ii), and (iii) of (6.11) (for the case $n=1$ ). By (6.10) we have $k \in \Phi_{\alpha \beta} J$ so that, again by $(6.10), k \prec_{\beta} k^{\prime}$ for some $k^{\prime} \in J^{*} \subseteq J$. But then, by (iii) of (6.11), $f \prec_{\alpha} k^{\prime}$ so that $f \in J$.

(6.13) If $\alpha<\beta$ and if $J \in \mathfrak{S}_{\beta}$, then $\Phi_{\alpha \beta} \Phi^{\alpha \beta} J=J$.

Proof. First let $f \in \Phi^{\alpha \beta} J$ so that $f \prec_{\alpha} \bigvee_{i=1}^{n} g_{i}$ for some $g_{1}, \cdots$, $g_{n} \in \bigcup\left\{I \in \mathfrak{S}_{\alpha} ; I \subseteq J\right\}$. By (6.11) there exist $k_{1}, \cdots, k_{n} \in \bigcup\left\{I \in \mathfrak{S}_{\alpha} ; I \subseteq J\right\}$ such that $f \prec_{\beta} \bigvee_{i=1}^{n} k_{i}$; hence $f \in J$. Thus $\Phi^{\alpha_{\beta}} J \subseteq J$ which clearly implies that $\Phi_{\alpha \beta} \Phi^{\alpha \beta} J \subseteq J$.

For the reverse inclusion, let $f \in J$ and let $f \prec_{\beta} g$ for some $g \in J$. Then there is an $h \in L$ such that $f \wedge h \leqq \beta$ and $g \vee h \geqq \eta>\beta$ for some $\eta \in Q$. Let $\beta<\beta_{1}<\beta_{2}<\eta<\beta_{3}$ in $Q$ and let $(k, l, m) \in \mathscr{S}\left(g, \alpha, \beta_{1}, \beta_{2}, \beta_{3}\right)$ so that $k \wedge l \leqq \alpha$ and $g \vee l \geqq \beta_{1}>\beta$. Now if $k_{1} \ll_{\alpha} k$, then $k_{1} \wedge l \leqq \alpha$ so that $k_{1} \prec_{\beta} g$ and hence $k_{1} \in J$. Thus $H=\left\{k_{1} \in L ; k_{1} \ll_{\alpha} k\right\}$ is an $S_{\alpha}$-ideal such that $H \subseteq \Phi^{\alpha \beta} J$. Therefore, by (6.10) and (6.12), $H \subseteq$ 
$\Phi_{\alpha \beta} H \subseteq \Phi_{\alpha \beta} \Phi^{\alpha \beta} J$. Next, by (6.5) there is a $k_{1} \in L$ such that $k_{1} \prec_{\alpha} k$ and $k \prec_{\beta} k_{1}$. Hence $k_{1} \in \Phi_{\alpha_{\beta}} \Phi^{\alpha \beta} J$. But, by (6.1), $k \vee h \geqq \eta$ so that, since $f \wedge h \leqq \beta$, we have $f \prec_{\beta} k$. Then $f \prec_{\beta} k_{1}$ so that $f \in \Phi_{\alpha \beta} \Phi^{\alpha \beta} J$. Thus $J \subseteq \Phi_{\alpha \beta} \Phi^{\alpha \beta} J$.

THEOREM 6.14. If $\alpha \leqq \beta$, then $\Phi_{\alpha \beta}$ is an isomorphism of the lattice $\mathfrak{S}_{\alpha}$ onto the lattice $\mathfrak{S}_{\beta}$ such that $\Phi_{\alpha \beta}^{-1}=\Phi^{v_{\beta}}$. Moreover, the restriction of $\Phi_{\alpha \beta}$ to $\mathfrak{M}_{\alpha}$ is a homeomorphism of $\mathfrak{M}_{\alpha}$ onto $\mathfrak{M}_{\beta}$ with the following property: For every $M_{\alpha} \in \mathfrak{M}_{\alpha}$ and every $M_{\beta} \in \mathfrak{M}_{\beta}, \Phi_{\alpha \beta} M_{\alpha}=M_{\beta}$ if and only if $M_{\alpha} \subseteq M_{\beta}$.

Proof. The theorem is obvious for $\alpha=\beta$. If $\alpha<\beta$, then the first statement is an immediate consequence of (6.12) and (6.13), and the second statement follows from the maximality of $M_{\alpha}$ and $M_{\beta}$, the final statement of (6.10), and the first statement of this theorem.

Now let $\mathfrak{M}(L)=\bigcup\left\{\mathfrak{M}_{\alpha} ; \alpha \in Q\right\}$. We define the relation $\sim$ on $\mathfrak{M}=$ $\mathfrak{M}(L)$ as follows: For each $M, N \in \mathfrak{M}$, we write $M \sim N$ in case $M \cap N \in \mathfrak{M}$.

(6.15) Let $\alpha \leqq \beta$, let $M \in \mathfrak{M}_{\alpha}$, and let $N \in \mathfrak{M}_{\beta}$. Then the following statements are equivalent:

(i) $M \sim N$;

(ii) $M \subseteq N$;

(iii) $\Phi_{\alpha \beta} M=N$.

Proof. The equivalence of (ii) and (iii) follows from Theorem 6.14. Moreover, (ii) clearly implies (i). To see that (i) implies (ii) suppose that $M \sim N$. Then $\alpha \in M \cap N$ while $\gamma \notin M \cap N$ whenever $\gamma>\alpha$, so that $M \cap N \in \mathfrak{M}_{\alpha}$. Thus by the maximality of $M$ and $M \cap N$ we conclude that $M=M \cap N \subseteq N$.

If $\alpha \leqq \beta \leqq \gamma$, and if $N \in \mathfrak{M}_{\beta}$, then by Theorem 6.14 there exists exactly one $M \in \mathfrak{M}_{x}$ and exactly one $P \in \mathfrak{M}_{\gamma}$ such that $M \subseteq N$ and $N \subseteq P$. Therefore we conclude from (6.15) that $\sim$ is an equivalence relation on $M$ such that the $\sim$-equivalence class, $M^{\sim}$, determined by $M \in \mathfrak{M}_{\alpha}$ is precisely the set of images of $M$ under all mappings $\Phi_{\alpha \beta}$ for $\alpha \leqq \beta$ and $\Phi^{\alpha \gamma}$ for $\alpha \geqq \gamma$.

We now agree that, for each $M \in \mathfrak{M}$ and each $\alpha \in Q, M_{\alpha}$ will denote the unique maximal $S_{\alpha}$-ideal $M^{\sim} \cap \mathfrak{M}_{\alpha}$ in $M^{\sim}$.

TheOREM 6.16. If for each $\alpha \in Q$ and each $f \in L$ we set

$$
U(f, \alpha)=\left\{M^{\sim} ; f \in M_{\alpha}\right\},
$$

then the family $\{U(f, \alpha) ; f \in L$ and $\alpha \in Q\}$ is an open base for a compact Hausdorff topology on

$$
X_{L}=\left\{M^{\sim} ; M \in \mathfrak{M}\right\} \text {. }
$$


Moreover, the mapping $\Theta_{\alpha}: M_{\alpha} \rightarrow M_{\alpha}{ }^{2}$ is a homeomorphism of $\mathfrak{M i}_{\alpha}$ onto $X_{L}$.

Proof. Suppose that $M^{\sim} \in U(f, \alpha) \cap U(g, \beta)$ and, say, $\alpha \leqq \beta$. Then $f \in M_{\alpha}, g \in M_{\beta}$, and $M_{\alpha} \subseteq M_{\beta}$. By (6.10) there is a $k \in L$ such that $k \in M_{\alpha}$ and $g \ll_{\beta} k$. Thus $g \ll_{\beta}(k \vee f)$ and $f \leqq(k \vee f)$ so that if $N^{\sim} \in U(k \vee f, \alpha)$, then $f \in N_{\alpha}$ and $g \in N_{\beta}$. That is, $U(k \vee f, \alpha) \subseteq$ $U(f, \alpha) \cap U(g, \beta)$. But $M^{\sim} \in U(k \vee f, \alpha)$; hence the family $\{U(f, \alpha)\}$ is an open base. Since, by Theorem $3.10, \mathfrak{M}_{x}$ is a compact Hausdorff space, the proof will be completed by showing that $\Theta_{\alpha}$ is a homeomorphism.

Let $\alpha \in Q$; then clearly the mapping $\Theta_{\alpha}$ is one-to-one and onto $X_{L}$. Consider a basic open set $U=U(f, \beta)$ in $X_{L}$. Let $\Psi$ be the homeomorphism $\Phi_{\alpha \beta}$ if $\alpha \leqq \beta$ or $\Phi^{\alpha \beta}$ if $\alpha>\beta$. Then $\Theta_{\alpha}^{-1}(U)=\left(\Theta_{\beta} \Psi\right)^{-1}(U)=$ $\Psi^{-1} \Theta_{\beta}^{-1}(U)$ is open in $\mathfrak{M}_{x}$ since, by Lemma $3.9, \Theta_{\beta}^{-1}(U)$ is open in $\mathfrak{M}_{\beta}$. Hence $\Theta_{\alpha}$ is continuous. Since $\mathfrak{M}_{\tau}$ is compact, it will suffice to show that $X_{L}$ is Hausdorff. Thus let $M^{\sim}, N^{\sim}$ be distinct elements of $X_{L}$. Then by Lemma 3.9 and the proof of Theorem 3.10 there exist $h, g \in L$ such that the open sets $\mathfrak{U}(h)=\left\{P \in \mathfrak{M}_{x} ; h \in P\right\}$ and $\mathfrak{M}(g)=\left\{P \in \mathfrak{M}_{\alpha} ; g \in P\right\}$ in $\mathfrak{M}_{\alpha}$ separate $M_{\alpha}$ and $N_{\alpha}$. Thus it is clear that $U(h, \alpha)$ and $U(g, \alpha)$ separate $M^{\sim}$ and $N^{\sim}$ in $X_{L}$.

7. Representations of $C$-lattices as function lattices. In this section we prove that if $L$ is a $C$-lattice relative to the separating chain $Q$, then $L$ is lattice-homomorphic [1] to a sublattice of $C\left(X_{L}, \bar{Q}\right)$. In particular, if $L$ is a $C_{1}$-lattice relative to $Q$ satisfying a suitable boundedness condition, then $L$ is isomorphic to a characterizing $Q$-sublattice of $C\left(X_{L}, \widehat{Q}\right)$. The latter result, together with Theorem 5.12 , provides a complete characterization of characterizing $Q$-sublattices.

Let $L$ be a $C$-lattice relative to the separating chain $Q$. We shall continue to use the conventions of $\S 6$ concerning lower case letters and concerning the labelling of the elements of the $\sim$-equivalence classes of $\mathfrak{M}=\mathfrak{M}(L)$ (see the paragraph preceding Theorem 6.16). Now for each $f \in L$ and each $M^{\sim} \in X_{L}$, set

$$
M(f)=\left\{\alpha \in Q ; f \in M_{\alpha}\right\} .
$$

Then for each $f \in L$ define $f^{*} \in F\left(X_{L}, \bar{Q}\right)$ by

$$
f^{*}\left(M^{\sim}\right)=\Lambda M(f)
$$

for all $M^{\sim} \in X_{L}$. Note that since $\bar{Q}$ is complete, $f^{*}$ is a well-defined element in $F\left(X_{L}, \bar{Q}\right)$.

LEMma 7.1. If $L$ is a C-lattice relative to the separating chain $Q$, then the mapping $f \rightarrow f^{*}$ is a lattice-homomorphism of $L$ onto a sub- 
lattice of $F\left(X_{L}, \bar{Q}\right)$.

Proof. Clearly if $f \leqq g$ in $L$ and if $M \in \mathfrak{M}$, then $M(g) \subseteq M(f)$; hence $f^{*} \leqq g^{*}$. Thus to complete the proof we need only verify that for every $f, g \in L, f^{*} \vee g^{*} \geqq(f \vee g)^{*}$ and $f^{*} \wedge g^{*} \leqq(f \wedge g)^{*}$. To prove the first of these inequalities, note that for every $M \in \mathfrak{M}$, if $f, g \in L$, then $M(f) \cap M(g) \subseteq M(f \vee g)$; hence $\left(f^{*} \vee g^{*}\right)\left(M^{\sim}\right)=[\Lambda M(f)] \vee[\Lambda M(g)]=$ $\Lambda[M(f) \cap M(g)] \geqq \Lambda[M(f \vee g)]=(f \vee g)^{*}\left(M^{\sim}\right)$. To prove the second, let $M^{\sim} \in X_{L}$ and suppose that $\alpha, \beta \in Q$ are such that $\alpha>\beta>(f \wedge g)^{*}\left(M^{\sim}\right)$. Then $f \wedge g \in M_{\beta}$ and $M_{\beta} \subseteq M_{\alpha}$. We shall prove that either $f \in M_{\alpha}$ or $g \in M_{\alpha}$. By (6.5) there exists $k \in L$ such that $k \prec_{\beta} g$ and $g \prec_{\alpha} k$. Since $f \wedge g \in M_{\beta}$, we have by Lemma 3.8 that either $f \in M_{\alpha}$ or $k \in M_{\beta}$. If $f \in M_{\beta}$, then $f \in M_{\alpha}$. If $k \in M_{\beta}$, then $k \in M_{\alpha}$, so that $g \prec_{\alpha} k$ implies $g \in M_{\alpha}$. Thus $\left(f^{*} \wedge g^{*}\right)\left(M^{\sim}\right) \leqq \alpha$ for every $\alpha>(f \wedge g)^{*}\left(M^{\sim}\right)$; hence $f^{*} \wedge g^{*} \leqq(f \wedge g)^{*}$.

THEOREM 7.2. If $L$ is a $C$-lattice relative to the separating chain $Q$, then the mapping $f \rightarrow f^{*}$ is a lattice-homomorphism of $L$ onto a sublattice of $C\left(X_{L}, \bar{Q}\right)$. Moreover, for each $\alpha \in Q, \alpha^{*}\left(M^{\sim}\right)=\alpha$ for all $M^{\sim} \in X_{L}$.

Proof. If $f \in L$, then $f^{*} \in C\left(X_{L}, \bar{Q}\right)$ provided that for every $\alpha \in Q$ the sets $\left\{M^{\sim} ; f^{*}\left(M^{\sim}\right)>\alpha\right\}$ and $\left\{M^{\sim} ; f^{*}\left(M^{\sim}\right)<\alpha\right\}$ are open. Thus let $\alpha \in Q$ and let $f^{*}\left(M^{\sim}\right)>\alpha$. Then for some $\gamma>\alpha, f \notin M_{\gamma}$. Let $\alpha<\beta<\gamma<\delta$ in $Q$, let $(k, l, m) \in \mathscr{S}(f, \alpha, \beta, \gamma, \delta)$, and let $\left(k^{\prime}, l^{\prime}, m^{\prime}\right) \in \mathscr{S}(k, \alpha, \beta, \gamma, \delta)$. Then, by (6.5) and (6.6), $f \prec_{\gamma} k \prec_{\gamma} k^{\prime}$ so that $k^{\prime} \notin M_{\gamma}$ and $k^{\prime} \notin M_{\alpha}$. But $k \wedge l \leqq \alpha$, and, by (6.6), $k^{\prime} \prec_{\alpha} k$ so that $l \in M_{\alpha}$ by Lemma 3.8 ; hence $M^{\sim} \in U(l, \alpha)$. Suppose that $N^{\sim} \in U(l, \alpha)$ and that $f^{*}\left(N^{\sim}\right) \leqq \alpha$. If $\alpha<\eta<\beta$, then $f, l \in N_{\eta}$. But $\beta \leqq f \vee l$, so that $\beta \in N_{\eta}$, contrary to $\eta<\beta$. Therefore $f^{*}\left(N^{\sim}\right)>\alpha$ for all $N^{\sim}$ in the open neighborhood $U(l, \alpha)$ of $M^{\sim}$.

To complete the proof let $f^{*}\left(M^{\sim}\right)<\alpha$. Then for some $\lambda<\alpha, f \in M_{\lambda}$. Let $\lambda<\eta<\zeta<\alpha$ and let $(k, l, m) \in \mathscr{P}(f, \lambda, \eta, \zeta, \alpha)$. Then $k \prec_{\lambda} f$, so that $k \in M_{\lambda}$ or, equivalently, $M^{\sim} \in U(k, \lambda)$. Suppose that $N^{\sim} \in U(k, \lambda)$ so that $k \in N_{\lambda}$. Then $k \in N_{\zeta}$, and since $f \prec_{\zeta} k$, we have $f \in N_{\zeta}$. Therefore $f^{*}\left(N^{\sim}\right) \leqq \zeta<\alpha$. That is, $f^{*}<\alpha$ on the open neighborhood $U(k, \lambda)$ of $M^{2}$.

Since the final statement of the theorem is obvious, the proof is complete.

Definition 7.3. Let $L$ be a $C$-lattice relative to the separating chain $Q$ and let

$$
L_{B}=\{f \in L ; \alpha \leqq f \leqq \beta \text { for some } \alpha, \beta \in Q\} .
$$


We call the lattice $L$ a bounded $C$-lattice in case $L=L_{B}$.

It follows readily that if $L$ is a $C$-lattice $\left(C_{1}\right.$-lattice) relative to $Q$, then so is $L_{B}$.

THEOREM 7.4. If $L$ is a C-lattice relative to the separating chain $Q$, then $X_{L}$ is a compact $\hat{Q}$-normal space and the mapping $f \rightarrow f^{*}$ is a lattice-homomorphism of $L_{B}$ onto a characterizing Q-sublattice of $C\left(X_{L}, \hat{Q}\right)$.

Proof. That $L_{B}$ is lattice-homomorphic, under $f \rightarrow f^{*}$, to a $Q$-sublattice of $C\left(X_{L}, \hat{Q}\right)$ follows from Theorem 7.2. To prove that the image of $L_{B}$ under this homomorphism is a characterizing sublattice of $C\left(X_{L}, \hat{Q}\right)$ it will suffice to prove that if $M^{\sim} \neq N^{\sim}$ in $X_{L}$, and if $\alpha, \beta \in Q$, then there exists an $f \in L_{B}$ such that $f \in M_{\alpha}$ and $f \notin N_{\beta}$. But $M_{\alpha} \nsim N_{\beta}$ implies $M_{\alpha} \nsubseteq N_{\beta}$, which in turn implies $M_{\alpha} \cap L_{B} \nsubseteq N_{\beta} \cap L_{B}$. To complete the proof, observe that $X_{L}$ is compact by Theorem 6.16 and is $\hat{Q}$-normal since $C\left(X_{L}, \hat{Q}\right)$ contains a characterizing $Q$-sublattice.

By Theorem 5.12 it is known that the image of $L_{B}$ under $f \rightarrow f^{*}$ is a $C_{1}$-lattice relative to $Q$ (identifying $Q$ with its image in $C\left(X_{L}, \hat{Q}\right)$ ). Moreover, it is obviously the case that if the mapping $f \rightarrow f^{*}$ is an isomorphism, then $L_{B}$ is itself a $C_{1}$-lattice relative to $Q$. Thus the existence of bounded $C$-lattices which are not $C_{1}$-lattices ( $c f$. Example 5.9) implies that, in general, the homomorphism $f \rightarrow f^{*}$ is not an isomorphism. However, if $L$ is a bounded $C_{1}$-lattice, then $f \rightarrow f^{*}$ is an isomorphism. To prove this we require the following lemma.

(7.5) Let $L$ be a distributive lattice, let $Q$ be a separating chain in $L$, and let $L$ satisfy (C.1) and (C.2') relative to $Q$. If $\alpha \in Q$ and if $f, g \in L$ with $f \geqq \alpha$ and $f \wedge g \leqq \alpha$, then $g \in M$ for some maximal $S_{\alpha}-$ ideal $M$ of $L$.

Proof. Since $L$ satisfies (C.1) and (C.2'), it is a $C$-lattice relative to $Q$. Since $f \not K \alpha$, there is a $\delta>\alpha$ such that $f \not \delta \delta$. Let $\alpha<\beta<\gamma<\delta$ in $Q$ and let $(k, l, m) \in \mathscr{S}(f, \alpha, \beta, \gamma, \delta)$. Set $I=\left\{h \in L ; h \ll_{\alpha} l\right\}$; then $I \in \subseteq_{\alpha}$. Since $f \wedge g \leqq \alpha$ and $f \vee l \geqq \beta>\alpha$, we have that $g \in I$. If $\beta \in I$, then from $l \wedge k \leqq \alpha$ it follows that $\beta \wedge k \leqq \alpha$. Then the inequalities $\beta \wedge k \leqq \alpha, m \vee k \geqq \delta, m \wedge f \leqq \gamma$, together with $\alpha \rightarrow \beta \rightarrow \gamma-\delta$, imply $f \leqq \gamma<\delta$, a contradiction. Therefore $\beta \notin I$, so that $I$ is a proper $S_{\alpha}$-ideal of $L$ with $g \in I$. A simple application of Zorn's lemma completes the proof.

THEOREM 7.6. If $L$ is a $C_{1}$-lattice relative to the separating chain $Q$, then $X_{L}$ is a compact $\hat{Q}$-normal space and the mapping $f \rightarrow f^{*}$ is 
an isomorphism of $L_{B}$ onto a characterizing $Q$-sublattice of $C\left(X_{L}, \hat{Q}\right)$.

Proof. In view of Theorem 7.4 it will suffice to prove that if $f \not 太 g$ in $L_{B}$, then $f^{*} \not g^{*}$ in $C\left(X_{L}, \hat{Q}\right)$. But if $f \not g g$, then, since $L_{B}$ is a $C_{1^{-}}$ lattice, there exists an $\alpha \in Q$ and there exists an $h \in L_{B}$ such that $h \wedge g \leqq \alpha$ and $h \wedge f \geqq \alpha$. Obviously we may assume that $h \leqq f$. Let $\alpha<\beta<\gamma<\delta$ in $Q$ with $h \not \Varangle \gamma$ and let $(k, l, m) \in \mathscr{S}(h, \alpha, \beta, \gamma, \delta)$. Suppose that $l \in M_{\alpha}$ for some $M_{\alpha} \in \mathfrak{M}_{\alpha}$. Then since $h \vee l \geqq \beta>\alpha$, we have that $g \prec_{\alpha} l$, and hence that $g \in M_{\alpha}$. Also if $\alpha<\eta<\beta$, then $l \in M_{\eta}$ so that $h \notin M_{\eta}$ and consequently $f \notin M_{\eta}$. Thus $g^{*}\left(M_{\alpha}^{-}\right) \leqq \alpha$ and $f^{*}\left(M_{\alpha}^{\sim}\right) \geqq \beta$. Therefore we need only prove the existence of some $M_{x} \in \mathfrak{M}_{x}$ with $l \in M_{\alpha}$. Since $k \wedge l \leqq \alpha$, it will suffice, in view of (7.5), to show that $k \npreceq \alpha$. But if $k \leqq \alpha$, then $k \vee m \geqq \delta$ and $\alpha-3 \delta$ imply that $m \geqq \delta$; therefore, since $m \wedge h \leqq \gamma$ and $\gamma-3 \delta$, we have $h \leqq \gamma$, a contradiction. Thus $k \npreceq \alpha$, as desired.

Clearly if $X$ is compact, then every characterizing $Q$-sublattice of $C(X, K)$ is bounded. Therefore, combining Theorem 4.3, Theorem 5.12, and Theorem 7.6, we have the following characterization.

THEOREM 7.7. Let $K$ be a conditionally complete and dense-in-itself chain with neither a first nor a last element, let $K$ be endowed with its interval topology, and let $Q$ be an order-dense subchain of $K$. If a lattice $L$ is isomorphic to a characterizing Q-sublattice of $C(X, K)$ for some compact space $X$, then the inverse image of $Q$ in $L$ is a separating chain in $L$ relative to which $L$ is a bounded $C_{1}$-lattice. Conversely, if $L$ is a bounded $C_{1}$-lattice relative to $Q$, then there exists an isomorphism of $L$ onto a characterizing $Q$-sublattice of $C(X, \hat{Q})$ for some topologically unique compact $\hat{Q}$-normal space $X$. Moreover, $X$ can be chosen as the space $X_{L}$ and the isomorphism can be chosen to be the mapping $f \rightarrow f^{*}$ so that the image of each $\alpha \in Q$ is the constant function $\alpha$ in $C\left(X_{L}, \hat{Q}\right)$.

8. Characterizations of the lattice $C(X, K)$. In this section we turn to the problem of obtaining necessary and sufficient conditions in order that a bounded $C_{1}$-lattice $L$ be isomorphic to the entire lattice $C(X, K)$ for some compact $K$-normal space $X$.

We give two solutions to this problem. The first is obtained by topologizing $L$ and then by employing an appropriate generalization (Lemma 8.2) of the Stone-Weierstrass theorem [16]. The second is obtained, without topologizing $L$, by a method which closely parallels that introduced by Fan [4] to characterize $C(X, R)$ as a partially ordered group.

As usual, let $Q$ be an order-dense subchain of $K$, and let $-\infty$ and 
$+\infty$ be the extreme elements of $\bar{K}$. For each finite subchain $\alpha_{1}<\cdots<\alpha_{n}$ of $Q$, let $\alpha_{0}=-\infty, \alpha_{n+1}=+\infty$, and let $\Gamma=\Gamma\left(\alpha_{1}, \cdots, \alpha_{n}\right)$ be the set of all intervals

$$
\left\{\gamma \in K ; \alpha_{i-1}<\gamma<\alpha_{i+1}\right\} \quad(i=1, \cdots, n) .
$$

We then set

$$
U(\Gamma)=\bigcup\{I \times I ; I \in I\} .
$$

It is readily verified that the family of all such sets $U(\Gamma)$ forms a base for a uniformity $\mathscr{C}$ on $K .^{9}$ Note that $\mathscr{U}$ is independent of the order dense subchain $Q$ of $K$.

If $L$ is a subset of $F(X, K)$, then we let $\mathscr{Z}(L)$ be the uniformity of uniform convergence induced on $L$ by $\mathscr{Z}[10$, p. 226].

Definition 8.1. Let $L$ be a $C_{1}$-lattice relative to the separating chain $Q$, and let $\mathscr{Y}$ be a uniformity on $L$. We shall say that $L$ is locally complete in the uniformity $\mathscr{Y}$ in case for each $\alpha<\beta$ in $Q$ the set

$$
L(\alpha, \beta)=\{f \in L ; \alpha \leqq f \leqq \beta\}
$$

is complete in $\mathscr{y}$.

Lemma 8.2. Let $X$ be a compact Hausdorff space and let $L$ be a characterizing $Q$-sublattice of $C(X, K)$. Then $L=C(X, K)$ if and only if $L$ is locally complete in the uniformity $\mathscr{Q}(L)$.

Proof. Since each closed and bounded interval in $K$ is complete in the uniformity $\mathscr{C}$, it follows that $C(X, K)$ is locally complete (see e.g. [10, p. 231]). Conversely, let $L$ be locally complete. Since each $f \in C(X, K)$ is bounded, it will suffice to prove that for each $\alpha<\beta$ in $Q$,

$$
L(\alpha, \beta)=\{f \in C(X, K) ; \alpha \leqq f \leqq \beta\} .
$$

The proof of this parallels that of the Stone-Weierstrass approximation theorem for lattices of real-valued continuous functions [16, Theorem 1]. Since the modifications required are slight, we omit the details.

Now let $L$ be a bounded $C_{1}$-lattice relative to the separating chain $Q$, and let $\mathscr{U}(L)$ be the family of all sets of the form

$$
\left\{(f, g) \in L \times L ;\left(f^{*}\left(M^{\sim}\right), g^{*}\left(M^{\sim}\right)\right) \in U \text { for all } M \in \mathfrak{M} \mathcal{Z}\right\}
$$

as $U$ ranges over $\mathscr{C}$. Since $f \rightarrow f^{*}$ is an isomorphism of $L$ onto a sublattice $L^{*}$ of $C\left(X_{L}, K\right)$, it is clear that $\mathscr{C}(L)$ is a uniformity on $L$ and

\footnotetext{
${ }^{9}$ In fact, $\mathscr{Q}$ is the uniformity determined by the finite normal coverings of $K$ [18].
} 
that $L$ and $L^{*}$ are uniformily equivalent.

Our first characterization of the entire lattice $C(X, K)$ is now an immediate consequence of Theorem 7.7, Lemma 8.2, and the above remarks.

THEOREM 8.3. If $L$ is a bounded $C_{1}$-lattice relative to the separating chain $Q$, and if $L$ is locally complete in the uniformity $\mathbb{Z}(L)$, then $X_{L}$ is a compact $\hat{Q}$-normal space and $L$ is lattice isomorphic to $C\left(X_{L}, \hat{Q}\right)$. Conversely, if $X$ is a compact $K$-normal space, and if $Q$ is an order-dense subchain of $K$, then $Q$ is a separating chain in $C(X, K)$ and, relative to $Q, C(X, K)$ is a bounded $C_{1}$-lattice locally complete in the uniformity $\mathscr{Q}(C(X, K))$.

A special case of this characterization is the following solution of Birkhoff's Problem 81.

CoRollary 8.4. A lattice $L$ is isomorphic to the lattice $C(X, R)$ for some compact Hausdorff space $X$ if and only if $L$ contains a countable separating chain $Q$ relative to which $L$ is a bounded $C_{1}$-lattice locally complete in the uniformity $\mathscr{U}(L)$.

Proof. The proof is an easy consequence of the fact that every compact Hausdorff space is $R$-normal and the fact that a countable chain without extreme points is dense-in-itself if and only if it is isomorphic to the chain of rational numbers [1, p. 31].

The next result is a lattice analogue of Fan's Lemma 8.1 [4].

LEMмA. 8.5. If $X$ is a compact space, if $L$ is a characterizing $Q$-sublattice of $C(X, K)$, and if there is an isomorphism $\Psi$ of $C(X, K)$ onto $L$ mapping $Q$ onto itself, then $L=C(X, K)$.

Proof. Since $Q$ is dense in $K$ and $K$ is conditionally complete, $\Psi$ maps $K$ onto itself, so that $L$ is, in fact, a characterizing $K$-sublattice of $C(X, K)$. Thus, by Theorem 5.12 , both $L$ and $C(X, K)$ are $C_{1}$-lattices relative to $K$. For each $x \in X$ and each $\alpha \in K$ we denote by $M_{\alpha}(x)$ (respectively, $\bar{M}_{\alpha}(x)$ ) the unique maximal $S_{\alpha}$-ideal of $C(X, K)$ (respectively, $L$ ) associated with $x$. Now let $\alpha \in K$. Then for each $x \in X$, Theorem 4.1 implies that

$$
\Psi M_{\alpha}(x)=\bar{M}_{\Psi x}(\Theta x)
$$

for some unique element $\Theta x \in X$, and, by Theorem 4.3,

$$
\Theta: x \rightarrow \Theta x
$$

is a homeomorphism of $X$. If also $\beta \in K$, then $\Psi M_{\beta}(x) \sim \Psi M_{x}(x)$, so 
that, by Theorem 4.1 and (6.15), $\Psi M_{\beta}(x)=\bar{M}_{\Psi \beta}(\Theta x)$. Thus $\Psi M_{\wedge}(x)=$ $\bar{M}_{\Psi_{\alpha}}(\Theta x)$ for every $x \in X$ and every $\alpha \in K$. It follows that for each $f \in C(X, K)$ and each $x \in X$,

$$
\begin{aligned}
f(x) & =\Lambda\left\{\alpha \in K ; f \in M_{v}(x)\right\} \\
& =\Lambda\left\{\alpha \in K ; \Psi f \in \bar{M}_{\Psi_{\alpha}}(\Theta x)\right\}
\end{aligned}
$$

and hence $\Psi[f(x)]=(\Psi f)(\Theta x)$. Suppose now that $f \in C(X, K)$ and define the function $g$ on $X$ by

$$
g(x)=\Psi^{-1}[f(\Theta x)] .
$$

Then clearly $g \in C(X, K)$. But for each $x \in X$,

$$
(\Psi g)(x)=\Psi\left[g\left(\Theta^{-1} x\right)\right]=\Psi\left[\Psi^{-1}\left[f\left(\Theta \Theta^{-1} x\right)\right]\right]=f(x) .
$$

Thus $f \in L$ and we conclude that $C(X, K)=L$.

Definition 8.6. Let $L$ be a $C_{1}$-lattice relative to the separating chain $Q$. Then a pair $\left(L^{\prime}, \Lambda\right)$ is an extension of $L$ in case $L^{\prime}$ is a lattice and $\Lambda$ is an isomorphism of $L$ into $L^{\prime}$ such that $L^{\prime}$ is a $C_{1}$-lattice relative to $1 Q$. The extension $\left(L^{\prime}, \Lambda\right)$ is said to be

(i) bounded in case, relative to $\Lambda Q, L^{\prime}$ is bounded;

(ii) direct in case for every pair $M, N$ in the set $M\left(L^{\prime}\right)$ of all maximal $S$-ideals of $L^{\prime}$, if $M \nsubseteq N$, then $M \cap \Lambda L \nsubseteq N$;

(iii) normal in case for every $f \in L^{\prime}$ and every $\alpha^{\prime}<\beta^{\prime}<\gamma^{\prime}<\delta^{\prime}$ in $\Lambda Q$, there exist $k, l, m \in \Lambda L$ such that $(k, l, m) \in \mathscr{S}_{\Lambda Q}^{L^{\prime}}\left(f, \alpha^{\prime}, \beta^{\prime}, \gamma^{\prime}, \delta^{\prime}\right)$.

The above notion of direct extension is patterned after Fan's notion of a direct extension of a partially ordered additive group [4, p. 411].

If $L$ is a $C_{1}$-lattice relative to $Q$ and if $\left(L^{\prime}, \Lambda\right)$ is an extension of $L$, then we shall henceforth make no notational distinction between the chain $Q$ in $L$ and the chain $A Q$ in $L^{\prime}$.

Lemma 8.7. If $L^{\prime}$ is a characterizing Q-sublattice of $C(X, K)$, if $L$ is a $Q$-sublattice of $L^{\prime}$, and if $I$ is the identity mapping of $L$ into $L^{\prime}$, then the following statements are equivalent:

(i) $L$ is a characterizing Q-sublattice of $C(X, K)$.

(ii) $\left(L^{\prime}, I\right)$ is a normal extension of $L$.

(iii) $\left(L^{\prime}, I\right)$ is a direct extension of $L$.

Proof. (i) $\rightarrow$ (ii). By Theorem 5.12, $L^{\prime}$ is a $C_{1}$-lattice relative to the separating chain $Q$. Thus $\left(L^{\prime}, I\right)$ is surely an extension of $L$. Since $L$ is characterizing, it is immediate from Lemma 5.6 that $\left(L^{\prime}, I\right)$ is a normal extension of $L$.

(ii) $\rightarrow$ (iii). Let $\mu, \nu \in K$, let $M_{\mu}$ be a maximal $S_{\mu}$-ideal of $L^{\prime}$, and 
let $M_{\nu}$ be a maximal $S_{\nu}$-ideal of $L^{\prime}$. By Theorem 4.1, $M_{\mu}=M_{\mu}(x)$ and $M_{\nu}=M_{\nu}(y)$ for some $x$ and $y$ in $X$. Let us suppose that $M_{\mu} \nsubseteq M$. If $x=y$, then $\nu<\mu$. Choose $\gamma \in Q$ such that $\nu<\gamma<\mu$. Then $\gamma \in M_{\mu} \cap L$ but $\gamma \notin M_{\nu}$ so that $M_{\mu} \cap L \nsubseteq M$. If $x \neq y$, choose $\alpha, \beta, \gamma, \delta \in Q$ such that $\alpha<\beta<\mu \wedge \nu$ and $\mu \vee \nu<\gamma<\delta$. Since $L^{\prime}$ is characterizing, there is an $f \in L^{\prime}$ such that $f(x)<\alpha$ and $f(y)>\delta$. Moreover, since $\left(L^{\prime}, I\right)$ is a normal extension of $L$, there exist $k, l, m \in L$ with $(k, l, m) \in \mathscr{S}_{Q}{ }^{\prime}(f, \alpha, \beta, \gamma, \delta)$. Then $f(x)<\alpha$ and $f \vee l \geqq \beta$ together imply that $l(x) \geqq \beta$; and then $l(x) \geqq \beta$ and $k \wedge l \leqq \alpha$ together imply that $k(x) \leqq \alpha<\mu$. In a similar manner we obtain $k(y) \geqq \delta>\nu$. Thus $k \in M_{\mu} \cap L$ but $k \notin M_{\nu}$ so that again $M_{\mu} \cap L \nsubseteq M_{\nu}$. The extension $\left(L^{\prime}, I\right)$ is therefore direct.

(iii) $\rightarrow$ (i). Let $x$ and $y$ be distinct points of $X$, let $f, g \in L$, and choose $\alpha, \beta \in Q$ such that $\alpha<f(x)$ and $\beta>g(y)$. Let $M_{\alpha}(x)$ be the maximal $S_{\alpha}$-ideal of $L^{\prime}$ associated with $x$ and let $M_{\beta}(y)$ be the maximal $S_{\beta}$-ideal of $L^{\prime}$ associated with $y$. Then $M_{\alpha}(x) \nsubseteq M_{\beta}(y)$ so that, since $\left(L^{\prime}, I\right)$ is direct, $M_{\alpha}(x) \cap L \nsubseteq M_{\beta}(y)$. There is therefore a function $h \in L$ such that $h(x) \leqq \alpha$ and $h(y) \geqq \beta$; that is, $L$ is characterizing. The proof of the lemma is now complete.

Lemma 8.8. Let $L$ be a bounded $C_{1}$-lattice relative to the separating chain $Q$, let $\left(L^{\prime}, \Lambda\right)$ be a bounded extension of $L$, and denote by $\Delta$ the isomorphism $f \rightarrow f^{*}$ of $L^{\prime}$ into $C\left(X_{L^{\prime}}, \hat{Q}\right)$. Then

(i) $\left(C\left(X_{L^{\prime}}, \hat{Q}\right), \Delta\right)$ is a bounded direct extension of $L^{\prime}$, and

(ii) the following assertions are equivalent: (1) $\triangle \Lambda L$ is a characterizing Q-sublattice of $C\left(X_{L^{\prime}}, \hat{Q}\right),(2)\left(L^{\prime}, A\right)$ is a normal extension of $L,(3)\left(L^{\wedge}, \Lambda\right)$ is a direct extension of $L$.

Proof. By Theorem 7.7, $X_{L^{\prime}}$ is compact and $\hat{Q}$-normal, and $\Delta L^{\prime}$ is a characterizing $Q$-sublattive of $C\left(X_{L^{\prime}}, \hat{Q}\right)$. By Theorem 5.12, $C\left(X_{L^{\prime}}, \hat{Q}\right)$ is a $C_{1}$-lattice relative to $Q$. Thus $\left(C\left(X_{L^{\prime}}, \hat{Q}\right), I\right)$ is a bounded extension of $\Delta L^{\prime}$; by Lemma 8.7 it is also a direct extension. Then it is immediate that $\left(C\left(X_{L^{\prime}}, \hat{Q}\right), \Delta\right)$ is a bounded direct extension of $L^{\prime}$. This establishes (i).

To prove (ii), observe that $\triangle A L$ is a $Q$-sublattice of the characterizing $Q$-sublattice $\Delta L^{\prime}$ of $C\left(X_{L^{\prime}}, \hat{Q}\right)$. Moreover, it is clear that $\left(L^{\prime}, \Lambda\right)$ is a normal (direct) extension of $L$ if and only if $\left(\Delta L^{\prime}, I\right)$ is a normal (direct) extension of $\triangle A L$. The equivalence of (1), (2), and (3) is therefore an immediate consequence of Lemma 8.7.

We can now state our second characterization of the lattice $C(X, K)$.

THeOREM 8.9. If $L$ is a bounded $C_{1}$-lattice relative to the separating chain $Q$, and if, for each bounded direct extension $\left(L^{\prime}, \Lambda\right)$ of $L, L$ 
is isomorphic to $L^{\prime}$, then $X_{L}$ is a compact $\hat{Q}$-normal space and $L$ is lattice isomorphic to $C\left(X_{L}, \hat{Q}\right)$. Conversely, if $X$ is a compact $K$-normal space and if $Q$ is an order-dense subchain of $K$, then $Q$ is a separating chain in $C(X, K)$ and, relative to $Q, C(X, K)$ is a bounded $C_{1}$-lattice with the property that if $\left(L^{\prime}, \Lambda\right)$ is a bounded direct extension of $C(X, K)$, then $A$ is an isomorphism of $C(X, K)$ onto $L^{\prime}$. Moreover, both of the preceding statements hold if "direct extension" is replaced by "normal extension".

Proof. Suppose first that $L$ satisfies the conditions of the first statement of the theorem. If we denote by $\Delta$ the isomorphism $f \rightarrow f^{*}$ of $L$ into $C\left(X_{L}, \hat{Q}\right)$, then, by Theorem 7.4 and Lemma 8.8(i), $X_{L}$ is a compact $\hat{Q}$-normal space and $\left(C\left(X_{L}, \hat{Q}\right), \Delta\right)$ is a bounded direct extension of $L$. Therefore, by hypothesis, $L$ is isomorphic to $C\left(X_{L}, \hat{Q}\right)$.

Conversely, suppose that $X$ is a compact $K$-normal space and that $Q$ is an order-dense subchain of $K$. Then, by Theorem $5.12, C(X, K)$ is a bounded $C_{1}$-lattice relative to the separating chain $Q$. Let $\left(L^{\prime}, \Lambda\right)$ be a bounded direct extension of $L=C(X, K)$ and denote by $\Delta$ the isomorphism $f \rightarrow f^{*}$ of $L^{\prime}$ into $C\left(X_{L^{\prime}}, \hat{Q}\right)$. Then, by Lemma 8.8 (ii), $\Delta \Lambda L$ is a characterizing sublattice of $C\left(X_{L^{\prime}}, \hat{Q}\right)$ so that, by Theorem $4.3, X$ is homeomorphic to $X_{L^{\prime}}$. Then $C\left(X_{L^{\prime}}, \hat{Q}\right)$ is isomorphic to $C(X, K)$ and hence also to $\triangle A L$. But then, by Lemma $8.5, \Delta \Lambda L=C\left(X_{L^{\prime}}, \hat{Q}\right)$. It follows that $\Lambda$ is an isomorphism of $C(X, K)$ onto $L^{\prime}$.

The final statement of the theorem is a consequence of the fact (Lemma 8.8 (ii)) that a bounded extension is direct if and only if it is normal. The proof is now complete

The preceding theorem yields a second solution of Birkhoff's Problem 81 (cf. the proof of Corollary 8.4).

Corollary 8.10. A lattice $L$ is isomorphic to the lattice $C(X, R)$ for some compact Hausdorff space $X$ if and only if (i) $L$ contains a countable separating chain $Q$ relative to which $L$ is a bounded $C_{1^{-}}$ lattice, and (ii) $L$ is isomorphic to $L^{\prime}$ for every bounded direct (normal) extension $\left(L^{\prime}, \Lambda\right)$ of $L$.

9. Remarks on earlier characterizations of $C(X, R)$. We now turn our attention to the case in which $K$ is the real chain $R$. In this final section we indicate briefly the relation between our results, particularly Theorem 7.7, and the known representation theorems for partially ordered groups ([15], [4], and [5]) and for translation lattices [9]. Since there clearly exist characterizing $Q$-sublattices of $C(X, R)$ which are neither groups nor translation lattices, Theorem 7.7 is not subsumed by these earlier results. 
Now let $G$ be a partially ordered commutative group satisfying the following conditions:

(i) $G$ contains a subgroup $Q$ order-isomorphic to an order-dense subgroup of the simply ordered group $R$ of real numbers.

(ii) $Q$ contains an archimedean element for $G$ (i.e., there is an $e \in Q$ such that if $f \in G$, then $n e \geqq f$ for some positive integer $n$ ).

(iii) If $f, g \in G$ and if $n f+g \geqq 0(n=1,2, \cdots)$, then $f \geqq 0$.

If $\bar{G}$ is the lattice-ordered group generated by $G$ in the completion of $G$ (cf. [5]), then it can be proved directly that $\bar{G}$ is a bounded $C_{1^{-}}$ lattice relative to the separating chain $Q$ in $\bar{G}$. Consequently, by Theorem 7.7, the mapping $f \rightarrow f^{*}$ maps $\bar{G}$ lattice isomorphically onto a characterizing $Q$-sublattice $\bar{G}^{*}$ of $C\left(X_{\bar{G}}, R\right)$. It is easily seen that $\bar{G}^{*}$ is a lattice-ordered subgroup of $C\left(X_{\bar{G}}, R\right)$ and that the mapping $f \rightarrow f^{*}$ preserves the group operations of $\bar{G}$. If $G$ is initially a lattice-ordered group, then $G=\bar{G}$; an application of Lemma 8.2 then yields Stone's characterization [15]. In general, since the image $G^{*}$ of $G$ in $\bar{G}^{*}$ is a partially ordered subgroup of $C\left(X_{\bar{G}}, R\right)$ which separates points in $X_{\bar{G}}$, we obtain Fan's characterization [4] by making use of a direct extension argument for partially ordered groups.

In this connection we observe that Fleischer [5] obtains a representation of a partially ordered group as a point separating group of continuous real-valued functions on a compact Hausdorff space from conditions (ii) and (iii) alone. This very general result ( $c f$. Problem II of the Introduction) apparently cannot be deduced from Theorem 7.7 since, for such a group, $\bar{G}$ need not satisfy (C.2) relative to any subchain; for example, let $G$ be the simply ordered group of integers.

It is easily seen that the real translates of any element in a translation lattice [9] form a separating chain in the lattice relative to which $\left(\mathrm{C} .2^{\prime}\right)$ is satisfied. However, simple examples of translation lattices can be found that fail to be characterizing sublattices for any compact space. $^{10}$ Consequently, translation lattices need not be $C_{1}$-lattices, and thus our results do not imply Kaplansky's.

Kaplansky points out that in order to obtain $C(X, R)$ from a subtranslation lattice an appropriate "stretching axiom" is required. Clearly condition (C.1) relative to some chain of translates provides such an axiom.

\section{REFERENCES}

1. G. Birkhoff, Lattice theory, Amer. Math. Soc. Colloquium Publications, 25, rev. ed., New York, 1948.

2. R. L. Blair, Stone's topology for a binary relation, Duke Math. J., 22 (1955), 271-280.

10 For example, let $L$ be the translation lattice of all real-valued functions on the discrete space $\{x, y\}$ such that $f(x) \leqq f(y)$. 
3. - A note on lattices of continuous functions. II. (Abstract) Bull. Amer. Math. Soc., 61 (1955), 565.

4. Ky Fan, Partially ordered additive groups of continuous functions, Ann. of Math., $\mathbf{5 1}$ (1950), 409-427.

5. I. Fleischer, Functional representation of partially ordered groups, Ann. of Math., 64 (1956), 260-263.

6. L. J. Heider, A characterization of function lattices, Duke Math. J., 23 (1956), 297301.

7. S. Kakutani, Concrete representation of abstract (M)-spaces, Ann. of Math., 42 (1941), 994-1024.

8. I. Kaplansky, Lattices of continuous functions, Bull. Amer. Math. Soc., 53 (1947), 617-623.

9. - Lattices of continuous functions II, Amer. J. Math., 70 (1948), 626-634.

10. J. L. Kelley, General topology, New York, 1955.

11. M. Krein and S. Krein, On an inner characteristic of the set of all continuous functions defined on a bicompacl Hausdor.ff space, C. R. (Doklady) Acad. Sci. URSS, 27 (1940), 427-430.

12. A. G. Pinsker, A lattice characterization of function spaces, (In Russian) Uspehi Mat. Nauk (N. S.), 12 (1957), 226-229.

13. T. Shirota, A generalization of a theorem of I. Kaplansky, Osaka Math. J., 4 (1952), 121-132.

14. M. H. Stone, A general thcory of spectra, I, Proc. Nat. Acad. Sci. U.S.A., 26 (1940), $280-283$.

15. , A general theory of spectra, II, Proc. Nat. Acad. Sci. U.S.A., 27 (1941), $83-87$.

16. - - The generalized Weierstrass approximation theorem, Math. Mag., 21 (1948), 167-184.

17. A. Tarski, Some notions and methods on the borderline of algebra and metamathematics, Proc. Int. Cong. Math. Cambridge, I (1950), 705-720.

18. J. W. Tukey, Convergence and uniformity in topology, Ann. of Math. Studies, 2 (1940).

\section{UNIVERSITY OF OREGON}




\section{PACIFIC JOURNAL OF MATHEMATICS}

\section{EDITORS}

\section{David Gilbarg}

Stanford University

Stanford, California

\section{R. A. Beaumont}

University of Washington

Seattle 5 , Washington

\author{
A. L. Whiteman
}

University of Southern California Los Angeles 7, California

L. J. Paige

University of California

Los Angeles 24, California

\author{
E. F. BECKENBACH \\ C. E. BURGESS \\ E. HEWITT \\ A. HORN
}

\author{
V. GANAPATHY IYER \\ R. D. JAMES \\ M. S. KNEBELMAN \\ L. NACHBIN
}

ASSOCIATE EDITORS
I. NIVEN

T. G. OSTROM

H. L. ROYDEN

M. M. SCHIFFER
E. G. STRAUS

G. SZEKERES

F. WOLF

K. YOSIDA

\section{SUPPORTING INSTITUTIONS}

\author{
UNIVERSITY OF BRITISH COLUMBIA \\ CALIFORNIA INSTITUTE OF TECHNOLOGY \\ UNIVERSITY OF CALIFORNIA \\ MONTANA STATE UNIVERSITY \\ UNIVERSITY OF NEVADA \\ OREGON STATE COLLEGE \\ UNIVERSITY OF OREGON \\ OSAKA UNIVERSITY \\ UNIVERSITY OF SOUTHERN CALIFORNIA
}

\author{
STANFORD UNIVERSITY \\ UNIVERSITY OF TOKYO \\ UNIVERSITY OF UTAH \\ WASHINGTON STATE COLLEGE \\ UNIVERSITY OF WASHINGTON \\ * * * \\ AMERICAN MATHEMATICAL SOCIETY \\ CALIFORNIA RESEARCH CORPORATION \\ HUGHES AIRCRAFT COMPANY \\ SPACE TECHNOLOGY LABORATORIES
}

Mathematical papers intended for publication in the Pacific Journal of Mathematics should be typewritten (double spaced), and the author should keep a complete copy. Manuscripts may be sent to any one of the four editors. All other communications to the editors should be addressed to the managing editor, L. J. Paige at the University of California, Los Angeles 24, California.

50 reprints per author of each article are furnished free of charge; additional copies may be obtained at cost in multiples of 50 .

The Pacific Journal of Mathematics is published quarterly, in March, June, September, and December. The price per volume (4 numbers) is $\$ 12.00$; single issues, $\$ 3.50$. Back numbers are available. Special price to individual faculty members of supporting institutions and to individual members of the American Mathematical Society: $\$ 4.00$ per volume; single issues, $\$ 1.25$.

Subscriptions, orders for back numbers, and changes of address should be sent to Pacific Journal of Mathematics, 2120 Oxford Street, Berkeley 4, California.

Printed at Kokusai Bunken Insatsusha (International Academic Printing Co., Ltd.), No. 6, 2-chome, Fujimi-cho, Chiyoda-ku, Tokyo, Japan.

PUBLISHED BY PACIFIC JOURNAL OF MATHEMATICS, A NON-PROFIT CORPORATION

The Supporting Institutions listed above contribute to the cost of publication of this Journal, but they are not owners or publishers and have no responsibility for its content or policies. 


\section{Pacific Journal of Mathematics}

\section{Vol. 9, No. $2 \quad$ June, 1959}

Lee William Anderson, On the breadth and co-dimension of a topological lattice

Frank W. Anderson and Robert L. Blair, Characterizations of certain lattices

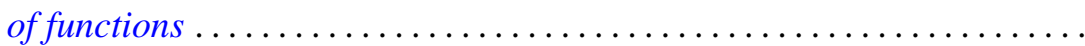

Donald Charles Benson, Extensions of a theorem of Loewner on integral

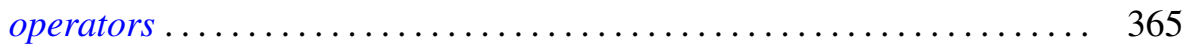

Errett Albert Bishop, A duality theorem for an arbitrary operator ........ 379

Robert McCallum Blumenthal and Ronald Kay Getoor, The asymptotic distribution of the eigenvalues for a class of Markov operators ........

Delmar L. Boyer and Elbert A. Walker, Almost locally pure Abelian

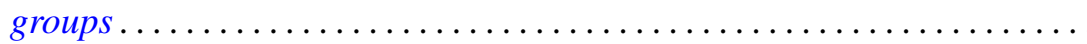

Paul Civin and Bertram Yood, Involutions on Banach algebras ........... Lincoln Kearney Durst, Exceptional real Lehmer sequences .... 415

Eldon Dyer and Allen Lowell Shields, Connectivity of topological

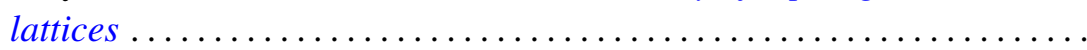

Ronald Kay Getoor, Markov operators and their associated

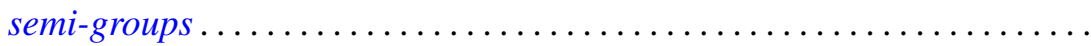

Bernard Greenspan, A bound for the orders of the components of a system of algebraic difference equations

Branko Grünbaum, On some covering and intersection properties in

Minkowski spaces ............................

Bruno Harris, Derivations of Jordan algebras ..............

Henry Berge Helson, Conjugate series in several variables.

Isidore Isaac Hirschman, Jr., A maximal problem in harmonic analysis.

II .

Alfred Horn and Robert Steinberg, Eigenvalues of the unitary part of a matrix

Edith Hirsch Luchins, On strictly semi-simple Banach algebras ...

William D. Munro, Some iterative methods for determining zeros of

functions of a complex variable...

John Rainwater, Spaces whose finest uniformity is metric .

William T. Reid, Variational aspects of generalized convex functions ....

A. Sade, Isomorphisme d'hypergroupoï des isotopes ...... . .

Isadore Manual Singer, The geometric interpretation of a special

connection . . .

Charles Andrew Swanson, Asymptotic perturbation series for characteristic

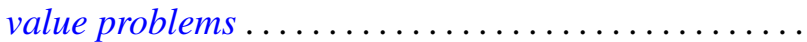

